\title{
Electronic Textiles: A Platform for Pervasive Computing
}

\author{
DIANA MARCULESCU, MEMBER, IEEE, RADU MARCULESCU, MEMBER, IEEE, \\ NICHOLAS H. ZAMORA, PHILLIP STANLEY-MARBELL, STUDENT MEMBER, IEEE, \\ PRADEEP K. KHOSLA, FELLOW, IEEE, SUNGMEE PARK, SUNDARESAN JAYARAMAN, \\ STEFAN JUNG, MEMBER, IEEE, CHRISTL LAUTERBACH, WERNER WEBER, SENIOR MEMBER, IEEE, \\ TÜNDE KIRSTEIN, DIDIER COTTET, MEMBER, IEEE, JANUSZ GRZYB, MEMBER, IEEE, \\ GERHARD TRÖSTER, MEMBER, IEEE, MARK JONES, MEMBER, IEEE, TOM MARTIN, MEMBER, IEEE, \\ AND ZAHI NAKAD, STUDENT MEMBER, IEEE
}

\section{Contributed Paper}

\begin{abstract}
The invention of the Jacquard weaving machine led to the concept of a stored "program" and "mechanized" binary information processing. This development served as the inspiration for C. Babbage's analytical engine-the precursor to the modern-day computer. Today, more than 200 years later, the link between textiles and computing is more realistic than ever. In this paper, we look at the synergistic relationship between textiles and computing and identify the need for their "integration" using tools provided by an emerging new field of research that combines the strengths and capabilities of electronics and textiles into one: electronic textiles, or e-textiles. E-textiles, also called smart fabrics, have not only "wearable" capabilities like any other garment, but
\end{abstract}

Manuscript received March 1, 2003; revised August 1, 2003. This work was supported in part by the Defense Advanced Research Projects Agency under Contracts F30602-00-2-0564, F30602-00-2-0548, DABT 63-96-C0083, and F33615-02-1-4004; in part by the U.S. Department of Navy under Contract N66001-96-C-8639; and in part by the Semiconductor Research Corporation under Contract 2001-HJ-898 and Grant 2002-RJ-1052G.

D. Marculescu, R. Marculescu, N. H. Zamora, P. Stanley-Marbell, and P. K. Khosla are with Department of Electrical and Computer Engineering, Carnegie Mellon University, Pittsburgh, PA 15213-3890 USA (e-mail: dianam@ece.cmu.edu; radum@ece.cmu.edu; nhz@ece.cmu.edu; pstanley@ece.cmu.edu; pkk@ece.cmu.edu).

S. Park and S. Jayaraman are with School of Polymer, Textile \& Fiber Engineering, Georgia Institute of Technology, Atlanta, GA 30332-0295 USA (e-mail: sungmee.park@ptfe.gatech.edu; sundaresan.jayaraman@ptfe.gatech.edu).

S. Jung, C. Lauterbach, and W. Weber are with Emerging Technologies, Corporate Research, Infineon Technologies AG, Munich, Germany (e-mail: stefan.jung@infineon.com; christl.lauterbach@infineon.com; werner.weber@infineon.com).

T. Kirstein, D. Cottet, J. Grzyb, and G. Tröster are with Wearable Computing Laboratory, Electronics Laboratory, Swiss Federal Institute of Technology, Zürich, Switzerland (e-mail: kirstein@ife.ee.ethz.ch; cottet@ife.ee.ethz.ch; jankiel@ife.ee.ethz.ch; troester@ife.ee.ethz.ch).

M. Jones, T. Martin, and Z. Nakad are with the Department of Electrical and Computer Engineering, Virginia Tech, Blacksburg, VA 24061-0111 USA (e-mail: mtj@vt.edu; tlmartin@vt.edu; znakad@vt.edu).

Digital Object Identifier 10.1109/JPROC.2003.819612 also have local monitoring and computation, as well as wireless communication capabilities. Sensors and simple computational elements are embedded in e-textiles, as well as built into yarns, with the goal of gathering sensitive information, monitoring vital statistics, and sending them remotely (possibly over a wireless channel) for further processing. The paper provides an overview of existing efforts and associated challenges in this area, while describing possible venues and opportunities for future research.

Keywords-Electronic textiles, fault tolerance, low-power design, remote execution, smart fabrics, wearable computing, wearable motherboard.

\section{INTRODUCTION}

J. Kay's invention of the flying shuttle in 1733 sparked the first Industrial Revolution, which led to the transformation of industry and subsequently of civilization itself. Yet another invention in the field of textiles-the Jacquard head by J.-M. Jacquard (circa 1801) — was the first binary information processor. At any given point, the thread in a woven fabric can be in one of two states or positions: on the face or on the back of the fabric. The cards were punched or cut according to the required fabric design. A hole in the card signified that the thread would appear on the face of the fabric, while a blank meant that the end would be left down and appear on the back of the fabric. The Jacquard head was used on the weaving loom or machine for raising and lowering the warp threads to form desired patterns based on the lifting plan or program embedded in the cards. Thus, the Jacquard mechanism set the stage for modern-day binary information processing. A. Lovelace, the benefactor for C. Babbage who worked on the analytical engine (the predecessor to the modern-day computer), is said to have re- 
marked, "The Analytical Engine weaves algebraic patterns just as the Jacquard loom weaves flowers and leaves." The Jacquard mechanism that inspired Babbage and spawned the Hollerith punched card has been instrumental in bringing about one of the most profound technological advancements known to humans, namely, the second Industrial Revolution also known as the Information Processing Revolution [1]. In fact, when Intel introduced its Pentium class of microprocessors, one of the advertisements had a "fabric of chips" emerging from a weaving machine; this picture eloquently captured the essence of chip making - a true blending of art and science-much like the design and production of textiles.

Nowadays, more than two centuries later, researchers are finding themselves looking at the possibility of integrating textiles and computing, but in a different way. Electronic textiles, or e-textiles, is an emerging interdisciplinary field of research that brings together specialists in information technology, microsystems, materials, and textiles. The focus of this new area is on developing the enabling technologies and fabrication techniques for the economical production of flexible, conformable and, optionally, large-area textile-based information systems that are expected to have unique applications for both civilian and military sectors.

The synergistic relationship between computing and textiles can also be characterized by specific challenges and needs that must be addressed in the context of electronic textile-based computing.

- The Three Dimensions of Clothing and Personalized Information Infrastructure: Humans are used to wearing clothes from the day they are born and, in general, no special "training" is required to wear them, i.e., to use the interface. In fact, it is probably the most universal of human-computer interfaces and is one that humans need, use, have familiarity with, and which can be easily customized [2]. Moreover, humans enjoy clothing and this universal interface of clothing can be "tailored" to fit the individual's preferences, needs, and tastes including body dimensions, budgets, occasions, and moods. Textiles can also be designed to accommodate the constraints imposed by the ambient environment in which the user interacts, i.e., different climates or operating requirements. In addition to these two dimensions of functionality (or protection) and aesthetics, if "intelligence" can be embedded or integrated into textiles as a third dimension, it would lead to the realization of clothing as a personalized and flexible wearable information infrastructure [3].

- Textiles and Information Processing: A well-designed information processing system should facilitate the access of information Anytime, Anyplace by Anyone-the three As. The "ultimate" information processing system should not only provide for large bandwidths, but also have the ability to see, feel, think, and act. In other words, the system should be totally "customizable" and be in tune with the human. Of course, clothing is probably the only element that is "always there" (and, thus, pervasive) and in complete harmony with the individual (at least in a civilized society). Also, textiles provide the ultimate flexibility in system design by virtue of the broad range of fibers, yarns, fabrics, and manufacturing techniques that can be deployed to create products for desired end-use applications. Moreover, fabrics provide "large" surface areas that may be needed for "hosting" the large numbers of sensors and processors that might be needed for deployment over large terrains, e.g., a battlefield. The opportunities to build in redundancies for fault tolerance make textiles an "ideal" platform for information processing.

- Need for Convergence: Today's consumer is demanding interactivity, connectivity, ease of use, and a "natural" interface for information processing. The enabling technologies of electronics, sensors, computing, and communications are becoming pervasive. Since textiles is pervasive-from clothes for newborns to senior citizens, from fabrics in automobile seats to tents in battlefields-and presents a "universal" interface [2], it has the potential to meet this emerging need of today's dynamic individual. Moreover, an individual is likely to be forgetful and leave a PDA behind, but is unlikely to walk out of the home without clothes. Therefore, there is a critical need to integrate the enabling technologies into textiles so that the traditionally passive, yet pervasive, textiles can be transformed into an interactive, intelligent information infrastructure for the demanding end user to facilitate pervasive information processing. Such a system can facilitate personalized mobile information processing (PMIP) and give new meaning to the term "human-machine symbiosis" in the context of pervasive/invisible computing [3].

This paper provides an overview of some of the existing efforts in this area, starting with one of the earliest accounts of on-fabric computing systems - the wearable motherboard developed at the Georgia Institute of Technology, Atlanta, in the mid-1990s (Section II). Section III introduces some of the challenges inherited from other ubiquitous computing environments, as well as some other ones, specific for e-textiles. Cutting-edge industrial techniques for integrating microelectronics in textiles are represented by Infineon's unique technology developed for a fabric-embedded MP3 player with voice control and capacitive sensing-based keypad (Section IV). Acknowledging the importance of textile-embedded interconnects, Section V presents a detailed analysis of signal transmission characteristics carried out at in the Wearable Computing Laboratory at the Swiss Institute of Technology, Zürich, Switzerland. Implications in failure modeling are also discussed here.

As a possible application for an e-textile-based service backplane, acoustic beamforming is described in Section VI, along with another possible application - the mapper garment, both developed at Virginia Tech, Blacksburg. Beamforming is also one of the driver applications for the dynamic power and fault-tolerance management developed at Carnegie Mellon University, Pittsburgh, PA. Such techniques are discussed in Section VII, which describes some 


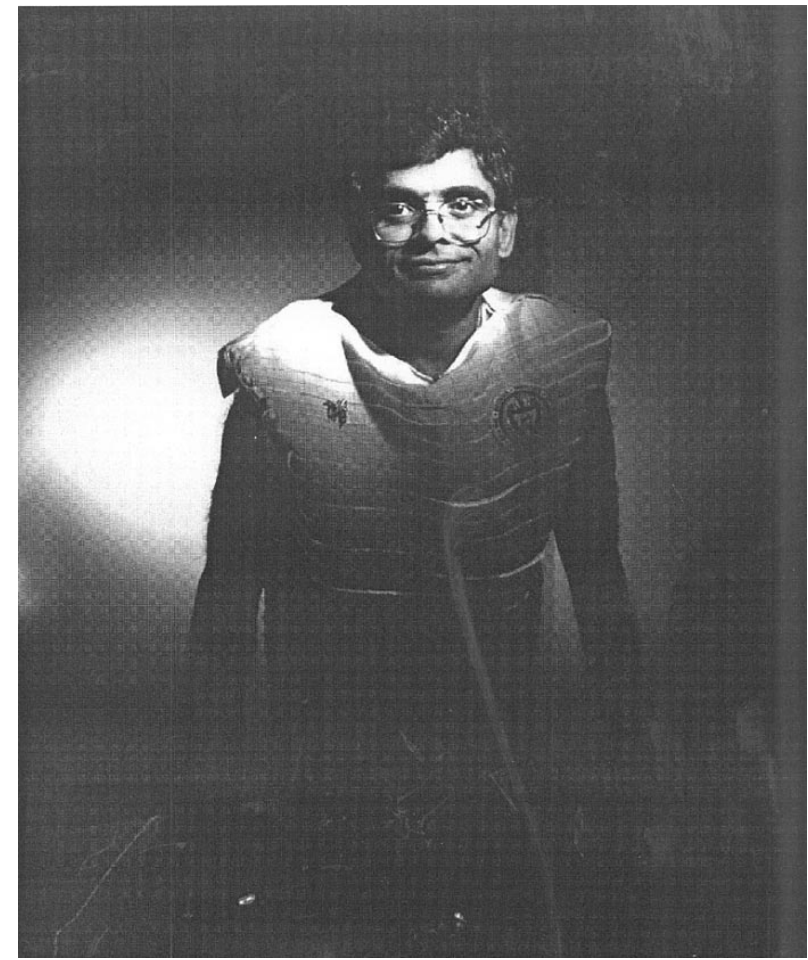

Fig. 1. The wearable motherboard (smart shirt).

of the existing, as well as novel techniques for prolonging lifetime of failure-prone e-textile-based applications. The paper concludes with a discussion of possible directions for future research and challenges related to this topic.

\section{The Early Days: The Georgia Tech Wearable MOTHERBOARD}

The design and realization of the Georgia Tech Wearable Motherboard (GTWM) has represented a significant advancement in the integration of textiles and computing paving the way for the paradigm of "fabric is the computer." Funded initially in October 1996 by the U.S. Department of the Navy, the GTWM (or the smart shirt) uses optical fibers to detect bullet wounds and special sensors and interconnects to monitor the body's vital signs during combat conditions [4]. However, as the research progressed, new vistas emerged for the deployment of the resulting technology (e.g., civilian applications) and the new paradigm of personalized information processing through a "wearable motherboard." Just as special-purpose chips and processors can be plugged into a computer motherboard to obtain the desired information processing capability, the smart shirt provides an extremely versatile framework for the incorporation of sensing, monitoring, and information processing devices. The principal advantage of the smart shirt is that it provides, for the first time, a very systematic way of monitoring the vital signs of humans in an unobtrusive manner.

Several generations of the woven and knitted versions of the the GTWM have been produced (see Fig. 1). The details of the design and development methodology have been discussed elsewhere [5]. Since the objective has been to create a comfortable and wearable information infrastructure, user requirements for the GTWM have been identified. These include factors such as wearability, durability, manufacturability, maintainability, connectivity, and affordability of the GTWM. For example, wearability implies that the GTWM should be lightweight, breathable, comfortable (form fitting), easy to wear and take off, and provide easy access to wounds. These are critical requirements in combat conditions so that the soldier's performance is not hampered by the protective garment. The durability of the GTWM is another important performance requirement. It should have a wear life of 120 combat days and should withstand repeated flexure and abrasion-both of which are characteristic of combat conditions. Manufacturability is another key requirement, since the design (garment) should be eventually produced in large quantities over the size range for the soldiers; moreover, it should be compatible with standard-issue clothing and equipment. Maintainability of the GTWM is an important requirement for the hygiene of the soldiers in combat conditions; it should withstand field laundering,dry easily, and be easily repairable (for minor damage). The developed GTWM should be easily connectable to sensors and the communications module on the soldier, known as the personal status monitor (PSM). Finally, affordability of the proposed GTWM is another major requirement so that the garment can be made widely available to all combat soldiers to help ensure their personal survival, thereby directly contributing to the military mission as force enhancers.

The developed interconnection technology has been used to integrate sensors for monitoring the following vital signs: heart rate, respiration rate, electrocardiogram (EKG), and pulse oximetry and temperature, among others. These sensors can be positioned anywhere on the body (locations being determined by a medical specialist) and are easily plugged into the smart shirt. The flexible data bus integrated into the structure routes the information from the sensors to a smart shirt controller that is seamlessly plugged into the shirt. The controller can then wirelessly transmit the monitored data to the desired display device, e.g., a wristwatch, PDA, a personal computer, or over the World Wide Web, using the appropriate communication protocol (Bluetooth, 802.11 b, etc.). The bus also carries information to the sensors (and, hence, the wearer) from external sources, thus making the smart shirt a valuable information infrastructure. The motherboard or "plug and play" concept means other sensors can be easily integrated into the structure. For instance, a sensor to detect oxygen levels or hazardous gases can be integrated into a variation of the smart shirt that will be used by firefighters. This information, along with the vital signs, can be transmitted wirelessly to the fire station, where personnel can continuously monitor the firefighter's condition and provide appropriate instructions including ordering the individual to evacuate the scene, if necessary.

Although begun as a "textile engineering" endeavor, the research has led to an even more groundbreaking contribution with significant implications: the creation of a wearable integrated information infrastructure that is lightweight, 
Table 1

The Wearable Motherboard/Smart Shirt: Potential Applications (From [2])

\begin{tabular}{|c|c|c|}
\hline Segment & Application Type & Target Audience \\
\hline Military & $\begin{array}{l}\text { Combat Casualty } \\
\text { Care }\end{array}$ & $\begin{array}{l}\text { Soldiers and Support Personnel } \\
\text { in Battlefield }\end{array}$ \\
\hline \multirow[t]{2}{*}{ Civilian } & Medical Monitoring & $\begin{array}{l}\text { Patients: Surgical Recovery, } \\
\text { Psychiatric Care } \\
\text { Senior Citizens: Geriatric } \\
\text { Care, Nursing Homes } \\
\text { Infants: SIDS prevention } \\
\text { Teaching Hospitals and } \\
\text { Medical Research Institutions }\end{array}$ \\
\hline & $\begin{array}{l}\text { Sports/Performance } \\
\text { Monitoring }\end{array}$ & $\begin{array}{l}\text { Athletes, Individuals } \\
\text { Scuba Divers, Mountaineers, } \\
\text { Hikers }\end{array}$ \\
\hline Space & Space Experiments & Astronauts \\
\hline Specialized & $\begin{array}{l}\text { Mission } \\
\text { Critical/Hazardous } \\
\text { Applications }\end{array}$ & Miners, Mass Transit Users \\
\hline $\begin{array}{l}\text { Public } \\
\text { Safety }\end{array}$ & $\begin{array}{l}\text { Fire-fighting } \\
\text { Law Enforcement }\end{array}$ & $\begin{array}{l}\text { Firefighters } \\
\text { Police }\end{array}$ \\
\hline Universal & $\begin{array}{l}\text { Wearable Mobile } \\
\text { Information } \\
\text { Infrastructure }\end{array}$ & $\begin{array}{l}\text { All Information Processing } \\
\text { Applications }\end{array}$ \\
\hline
\end{tabular}

comfortable, and launderable, and which has opened up new frontiers in personalized information processing, health care, and space exploration, to name a few [6].

Table 1 summarizes the broad range of applications of the smart shirt technology (a detailed discussion of the benefits of the technology in healthcare applications can be found in [2] and [7]). Just as the spreadsheet pioneered the field of information processing that brought "computing to the masses," it is anticipated that the GTWM will bring personalized and affordable health care monitoring to the population at large.

To demonstrate the feasibility of the wearable motherboard paradigm [8], [9], an "in-fabric" network using field-programmable gate arrays (FPGAs) and "soft" interconnects has been created. The chosen FPGAs were physically integrated into the fabric (see Fig. 2). Software was developed to demonstrate the "in-fabric" network. One of the FPGAs communicated with an external agent (a Linux-based personal computer) that was responsible for managing the global configuration of the FPGAs in the fabric by sequencing the "discovery" in the fabric beginning with that initial FPGA.

Two software modules were created. The first was developed to "demonstrate" the pin-connection discovery algorithm implemented in the system for identifying the connections between the various pins on the FPGAs in the fabric and to display the connection paths. This enables discovery of interconnects "on the fly" after the manufacturing has been carried out, under no a priori knowledge of the specific connections between the elements in the fabric. The second module discovers the connections and displays the paths on the screen as the discovery process proceeds when the FPGA is powered. To demonstrate the flow of information in the fabric network through the soft interconnects, a potentiometer was attached as a daughterboard to one of the FPGAs and whenever it was "twiddled," the resulting change-from the steady state-was displayed on the screen. The potentiometer is one example of an "external" device that can be attached to the fabric network through the FPGA. Depending on the intended application, specific sensors, effectors, and communications devices could be attached as daughtercards to the FPGAs to realize the desired functionality-e.g., vital signs monitoring-in the fabric.

Thus, the wearable motherboard fulfills the twin roles of being: 1) a flexible information infrastructure that will facilitate the paradigm of ubiquitous or pervasive computing and 2) a system for monitoring the vital signs of individuals in an efficient and cost-effective manner with a "universal" interface of clothing. This "fabric is the computer" paradigm exemplified by the GTWM demonstrates the feasibility of realizing PMIP and sets the stage for e-textiles.

\section{THE E-TEXTILES VISION}

Fig. 3 depicts the vision for "e-textiles" or the paradigm of "fabric is the computer" [10]. The major facets illustrate the various building blocks of the system that must be seamlessly integrated to realize the vision, starting with the underlying physical fabric or platform.

The design of this platform or infrastructure involves the exploration of materials, structures, and manufacturing technologies. The second key facet for realizing this paradigm of a true computational fabric is the interconnect architecture in the fabric, which involves the design and incorporation of physical data paths and interconnection technologies, i.e., the realization of "textile electrical circuits." Integration of sensors, microchips, and other devices (e.g., for communication and control) is critical for the realization of an "intelligent" e-textiles for any application, for example, battlefield management; therefore, hardware integration constitutes the third facet or building block shown in Fig. 3. Issues related to information processing such as fault tolerance in light of manufacturing defects and quality of service (QoS) within the e-textile and between the e-textile and external agents/devices are critical for the incorporation and optimal utilization of computing resources;therefore, software is the fourth facet of the e-textile continuum. And finally, as shown in the figure, a set of underlying performance metrics ranging from the physical dimensions (of the resulting structure/system) to costs, manufacturability, and dataflow rates must be utilized to assess the successful realization and performance of the desired e-textile. Thus this paradigm of "fabric is the computer" represents a fascinating area of research that fosters collaboration among scientists and engineers from a variety of disciplines including textiles, computing and communications, sensor technologies and application domains (e.g., medicine, space, military).

There have been a handful of attempts to design and build prototype computational textiles. In one of the earliest accounts [11], the authors demonstrate attaching off-the-shelf electrical components such as microcontrollers, surface 


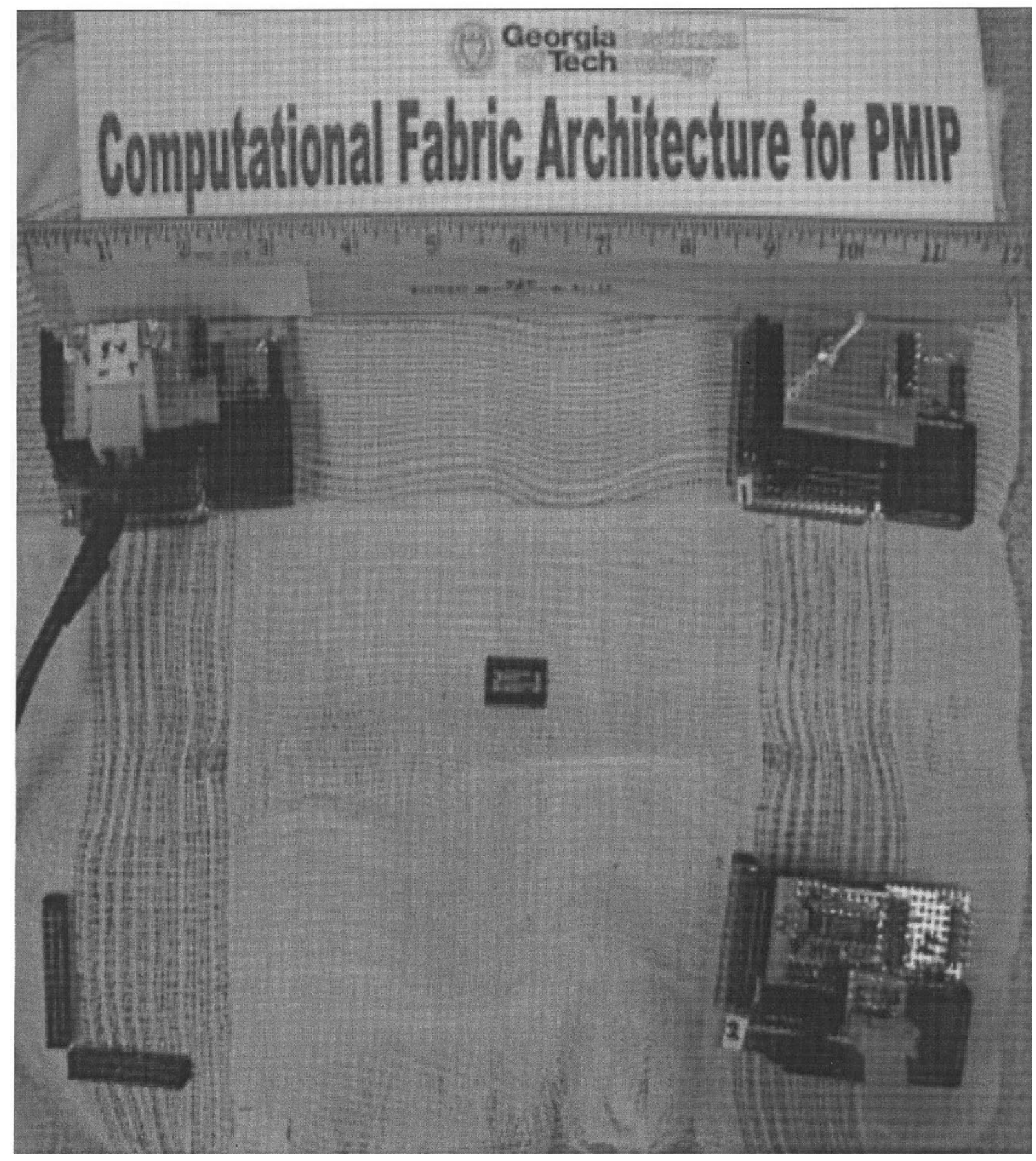

Fig. 2. The PMIP network in a fabric.

mount LEDs, piezoelectric transducers, etc., to traditional clothing material, transforming the cloth into a breadboard of sorts. In fabrics that contain conductive strands, these may be used to provide power to the devices, as well as to facilitate communication between devices. In [12], the authors extend the work presented in [11], detailing methods by which items such as user interfaces (keypads) and even chip packages may be constructed directly by a textile process. The routing of electrical power and communications, through a wearable fabric, was addressed in [13], where the authors provide a detailed account of physical and electrical components for routing electricity through suspenders made of a fabric with embedded conductive strands. Complete apparel with embedded computing elements is described in
[14]. The authors describe a jacket designed for the arctic environment, which augments the capabilities of the wearer with a global positioning system, sensors (accelerometers, conductivity electrode, heart rate monitors, and digital thermometers) and heating. However, all these approaches are single design points and do not provide a methodology for the evaluation and validation of e-textile systems.

It is our belief that in support of e-textiles a new design paradigm is needed, as well as a more general model of computation, which supports local computation and inexpensive communication among computational elements [15]-[17]. In the classic design cycle [Fig. 4(a)], the application is mapped onto a given platform architecture, under specified constraints (performance, area, power consumption). When 


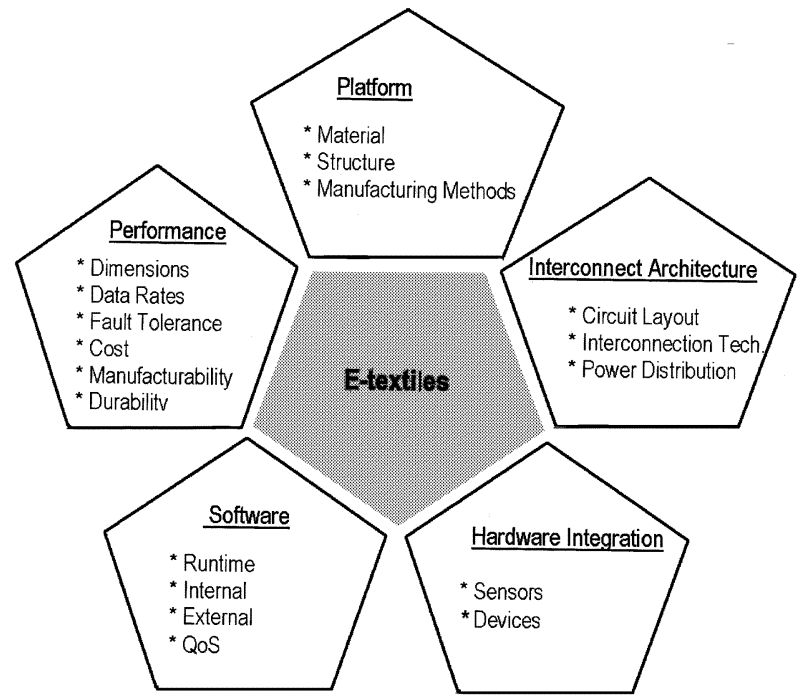

Fig. 3. The e-textiles Vision (from [10]).

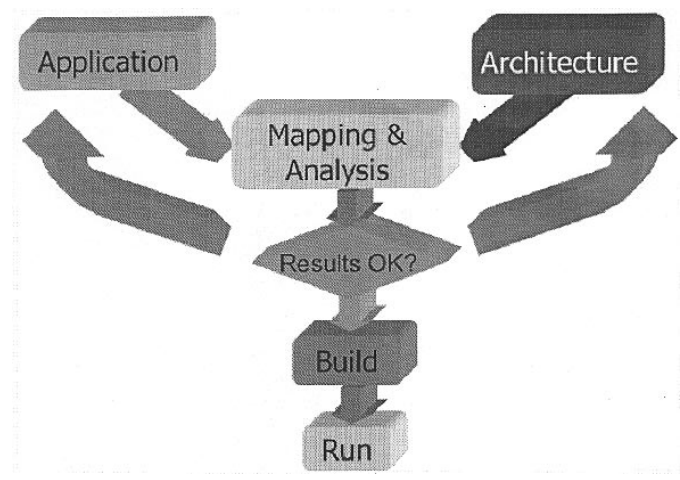

(a)

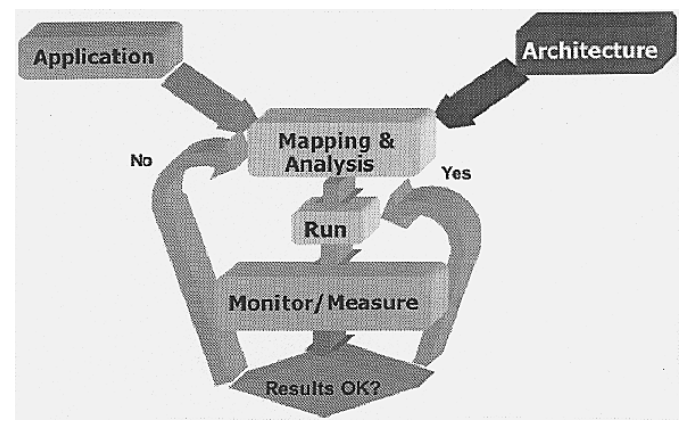

(b)

Fig. 4. (a) Classic design cycle versus (b) e-textile lifetime cycle.

these constraints are met, the prototype is tested, manufactured, and used for running the application. In the case of e-textiles [Fig. 4(b)], wide area textile networks are built onto e-textiles, with no prescribed functionality. To achieve high yields (that is, low defect rate), as well as high fault tolerance later in the lifetime cycle, regularity is important. The application is modified so as to expose as much local computation as possible, via partitioning or refinement. At power-up, the application is mapped so as to optimize different metrics of interest (such as quality of results, power consumption, operational longevity, and fault tolerance) and later remapped whenever operating conditions change.
Although e-textiles ostensibly present distributive computing challenges similar to those currently being pursued in adaptive control network and silicon-based pervasive computing systems, the specific characteristics and demands of an e-textiles info-computing environment add new dimensions to those challenges. In fact, e-textiles impose specific challenges as opposed to other applications in the general area of networked systems.

\section{A. What E-Textiles Are Not}

- Classical data networks. While the underlying structure of an e-textile application implies the existence of many processing elements, connected in a textile-area network (TAN), they have limited processing and storage capabilities, as well as very limited power consumption budgets. Hence, classic techniques and inherent methodologies for coping with mapping an application, communication among nodes, and dealing with network failures (including congestion) are not appropriate. In addition, having very limited processing capabilities, e-textiles are not the equivalent of "desktops/laptops on a fabric," restricting significantly the range of applications that can be mapped on them.

- Networked embedded systems (including wireless sensor networks). Wireless sensor networks share with e-textile-based applications the limited power budgets and, somewhat, the limited processing and storage capabilities. However, communication among processing elements is wired, and, thus, much more power efficient than the wireless communication. In addition, as opposed to ad hoc networks, the topological location of different processing elements is fixed throughout the lifetime of the application (although the mapping of the application on processing elements may change). Lastly, e-textiles must have low manufacturing costs; thus, the defect rate of the processing nodes (and physical links) will be much higher and very different than in the case of wireless networks. More limited processing and storage capabilities in conjunction with higher failure rates imply that existing body of research for sensor networks is not applicable directly to TANs.

\section{B. What E-Textiles Are}

E-textiles are "living designs," consisting of low-cost, simple components and interconnect, that are possibly unreliable or failure prone due to their operating environment. These characteristics are in fact shared by other nonsilicon computing systems (such as those based on nanoelectronics). However, in many ways, e-textiles systems may be considered the conformable, fabric equivalent of rigid body, embedded microelectromechanical systems (MEMS). However, unlike MEMS, e-textiles require coping with tears in the fabric, whether it is due to regular wear of the fabric 


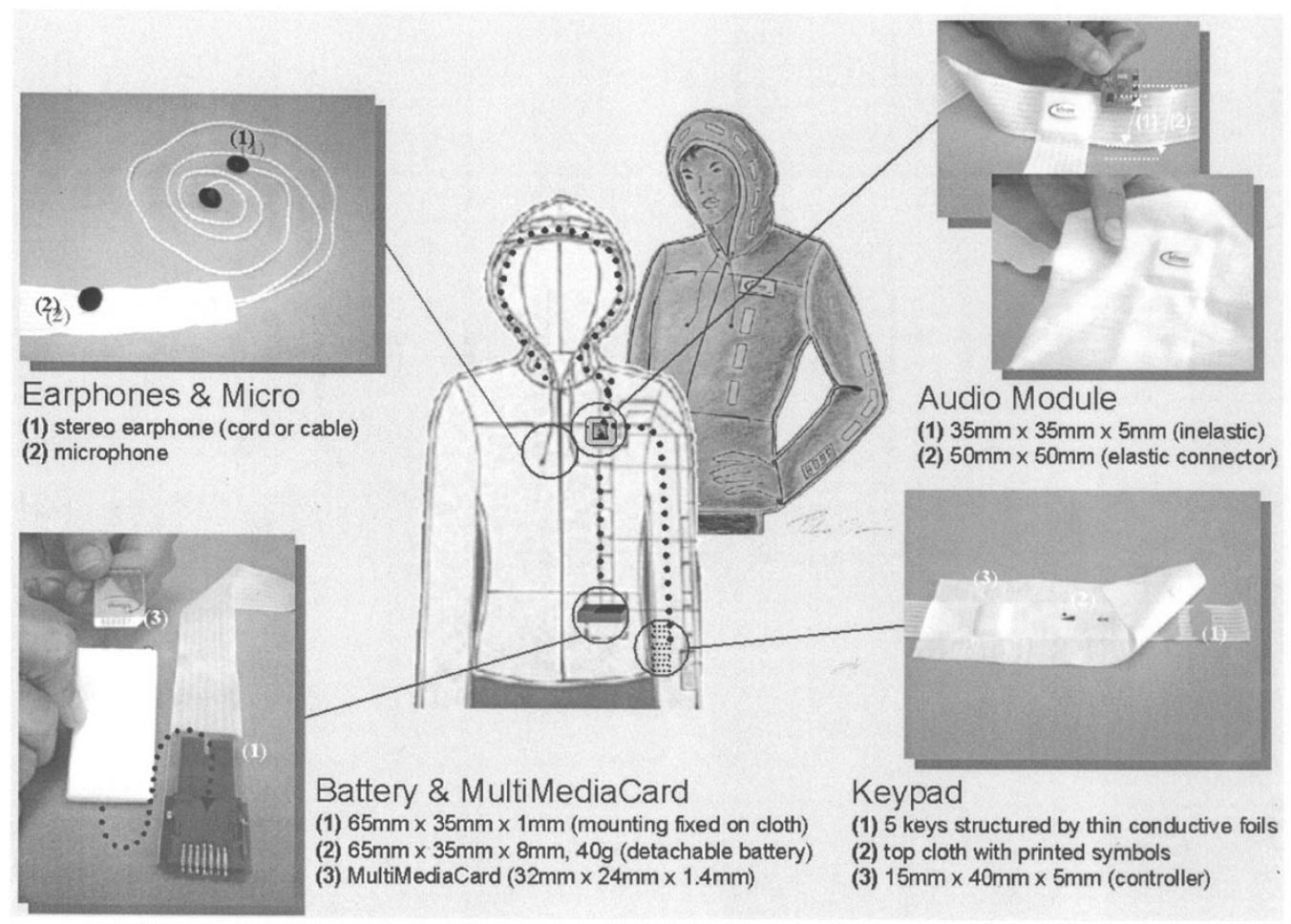

Fig. 5. Overview of the digital music player system with the proposed textile design-in. The dotted lines indicate the textile wiring realized by a narrow fabric with conductive stripes.

or to unexpected damage. The salient features of e-textiles are:

- limited processing, storage; and energy per computational node;

- potential failures for both nodes and communication links;

- highly spatially and temporally correlated node and/or link failures due to topological placement or due to external events;

- need for scalability and flexibility of resource management, as well as local versus global management tradeoff;

- active or smart links which embed simple logic (passive or active components).

In the following, we provide an overview of some of the key research efforts in support of the paradigm of e-textilebased computing. These efforts provide a vertical cut across all layers of interest, from embedding microelectronics or interconnect into fabric to providing system-level support for dynamic power and fault-tolerance management via lightweight middleware.

\section{INTEGRATED MicROELECTRONICS FOR SMART TEXTILES}

For integration into everyday clothing, electronic components should be designed in a functional, unobtrusive, robust, small, and inexpensive way [18]-[21]. Therefore, small single-chip microelectronic systems rather than large-scale computer boxes are a promising approach.
Today, a number of entertainment, safety, and communication applications have become possible candidates for deploying onto clothing using off-the-shelf chip systems. Such examples include audio processing chips for MP3 ${ }^{1}$ music, fingerprint sensor chips for person identification, Bluetooth chipsets for short-range wireless data exchange, or smart electronic labels for logistics purposes [22]. With the ongoing progress of miniaturization, many complex and large electronic systems will soon be replaced by tiny silicon microchips measuring just a few square millimeters.

In this section, we demonstrate the implementation of microelectronic components into clothes and textile structures in a reliable and manufacturable way. The example presented is a speech-controlled digital music player system [23]. Attention has been paid to an appropriate textile design for the tailoring of smart clothes. Damage of the components by washing processes and daily use must be avoided. Most solutions known so far require removal of complex electronics before starting the cleaning process. Our aim has been to demonstrate technological solutions for the interconnect between textile structures and electronics and for a robust electronic packaging which does not impair wearing comfort and offers ease of use to the customer.

\section{A. Demonstrator System Overview}

Fig. 5 gives an overview of the demonstrator system. It is composed of four units: the central audio module, a

${ }^{1} \mathrm{MP} 3$ is a digital data format, which allows for a compressed storage of high-quality music and audio data. 
detachable battery and MultiMediaCard (MMC) module, an earphone and microphone module, and a flexible keyboard sensor module. All units are electrically connected via ribbon-like narrow fabrics with conductive threads.

The core of the digital music player is the audio module. The module contains an audio signal processing microchip, which can be programmed to perform various functions, as discussed later. Besides this chip, further components such as program memory and a few auxiliary devices are implemented. All together, the module measures approximately $25 \times 25 \times 3 \mathrm{~mm}^{3}$. In principle, the audio module is a simple miniaturized computer system. Microphones, earphones, storage media, keypads, displays, sensors, actuators, and a battery can be directly connected to its interfaces. The functionality of the module is determined by the built-in software. Speech recognition, MP3 decoding, text-to-speech conversion, and music synthesis are only a few possible applications. An overview on the technical schematic and the existing software of the core microchip has been presented in [24].

The functionality of this main module is tailored for the typical requirements in wearable electronics and smart clothes, since speech recognition allows for hands-free interaction with the system. The system can be extended to establish a connection to standard networks like the Internet (software modem function).

The detachable battery and MMC module features a rechargeable lithium-ion polymer battery to supply the necessary electric energy. Its capacity is sufficient for an operating time of several hours. The battery module weighs only $50 \mathrm{~g}$ and is fixed to the cloth by means of a simple one-piece connector. A slot for the MMC has been integrated into the housing of the battery module. Both battery and MMC can thus be detached fast and easily. The MMC stores up to $64 \mathrm{Mb}$ of digital music or audio data. When detached from the cloth, the battery and MMC module can be plugged to a PC in order to recharge the battery and download new music to the MMC. The MMC is a standardized memory card, which can also be used with many other digital consumer electronics products, such as digital cameras, PDAs, or mobile phones.

The flexible keyboard sensor module consists of thin metallic foils brought onto the conductive narrow fabric. The metal foils are fixed using melting adhesives commonly used in garment production. The metal foils are connected to a small sensor chip module that detects whether a finger is close to a specific pad or not. By means of the keys, the user can activate the music player, control the volume or activate voice control. Voice recognition (“Stop!”; "Start!”; "Louder!") is activated after a specific button of the keypad has been touched. This measure avoids unintentional activation of control functions. The audio module recognizes the spoken words, e.g., the number or title of the music track. The earphone and microphone module simply consists of a piece of narrow fabric connecting the audio module and conventional earpieces and microphones, as depicted in Fig. 5.
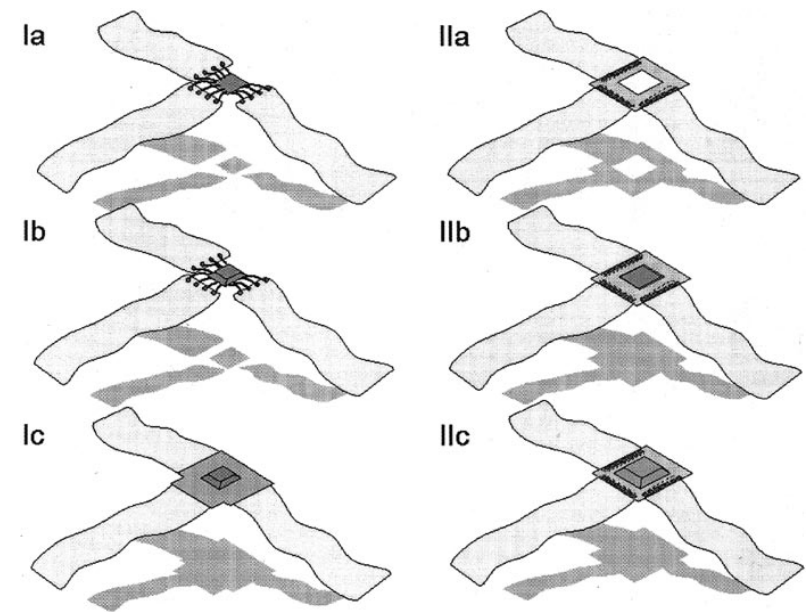

Fig. 6. Two interconnect concepts for the audio module. The module can be wire bonded to the narrow fabric (Ia-c) or connected to a thin flexible plastic foil with patterned interconnects (IIa-c). In both cases, the module and the interconnect areas are fully encapsulated to ensure stability against mechanical and leakage problems (steps lc and llc, respectively).

Special care is necessary for the textile design. All materials are chosen according to maximum wear comfort and environmental compliance. For example, the audio module is fully covered by garment. The wearer still has a comfortable textile touch in case the electronic module gets into direct contact with the skin. The supply voltages of the integrated electronics are as harmless as of a standard Walkman or comparable device.

\section{B. Interconnect Technology Between Textiles and Electronics}

Recent advances in microelectronics have enabled the manufacturing of integrated electronic circuits with millions of logic switching elements per square millimeter of silicon. Since the feature sizes of these devices are in the micrometer regime and the typical dimensions in textile and garment technologies are in the order of several millimeters, a novel technology for the electrical interconnects has been developed.

The gap of the spatial dimensions can be overcome by two methods that are described in the following. For test purposes, a polyester narrow fabric with several groups of parallel conductive warp threads has been used. In a first approach (Fig. 6, Ia-Ic), the endings of the conductive fabric are prepared by soldering tiny metal contact plates. The module is then connected by electrically isolated bonding wires. In a last step, the module, the wires, and the contact plates are molded for mechanical and chemical protection. A second approach (Fig. 6, IIa-IIc) uses a thin flexible circuit board with structured electrodes, which is glued and soldered to the textile structure before being molded.

Copper wires qualified for textile applications coated with silver and polyester have been used for the experiments. Fig. 7 shows microphotographs of the interconnect areas. The electrical isolation of the wires is removed by laser treatment [Fig. 7(a)]. The resulting holes of the fabric are 

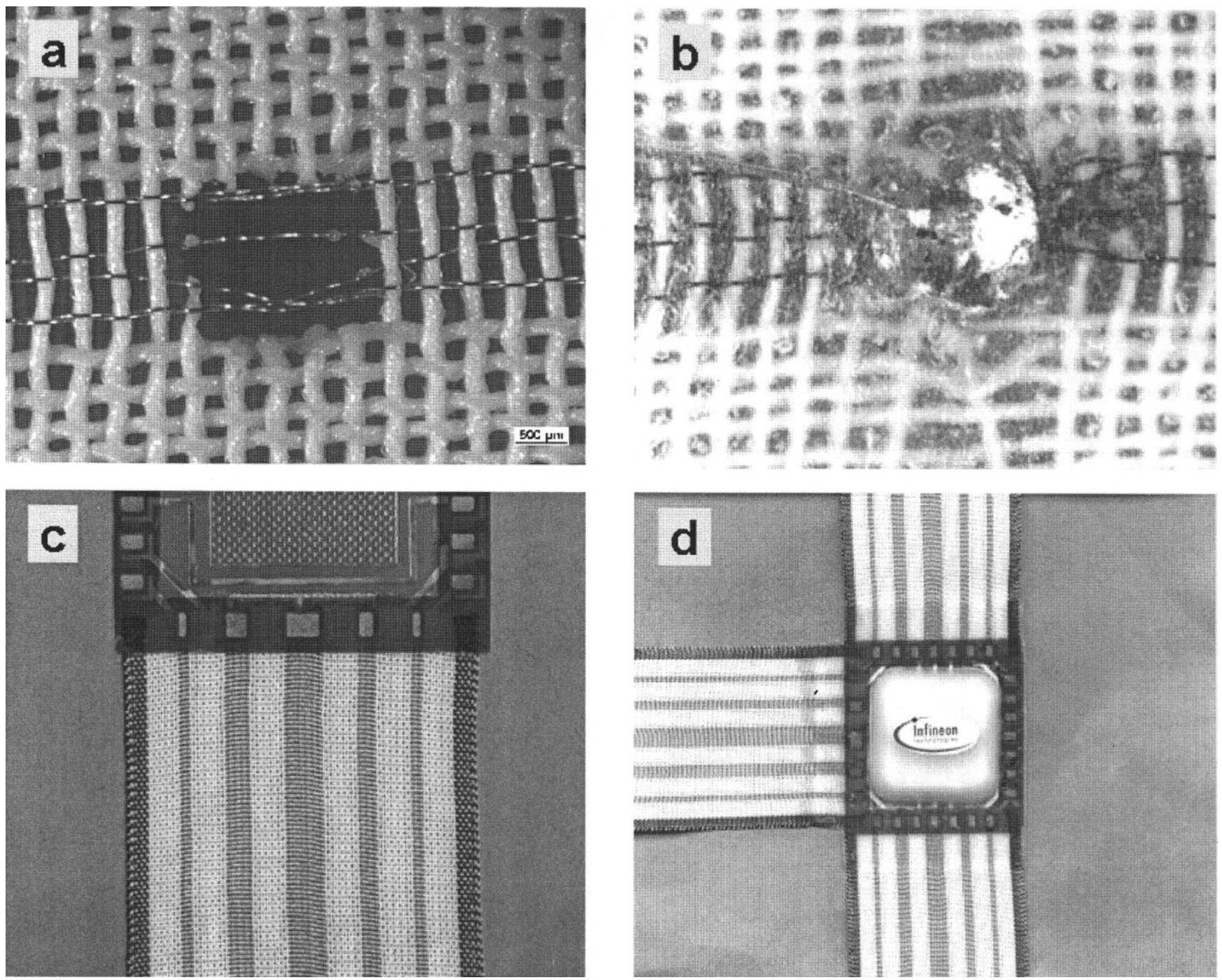

Fig. 7. Microphotographs of interconnect experiments performed on a woven test ribbon. (a) The coating of the wires is removed by laser treatment. (b) The woven wires are soldered to a small metal foil and connected to an electronic circuit by a thin wire. (c) Alternatively, the contact to an electronic module can be established via a flexible circuit board soldered to the ribbon. (d) Finally, the module and the contact areas are molded.

then filled with tiny metal plates of the same size. These connect the woven wires by soldering. A mechanical connection between metal and the fabric is achieved by melting and resolidifying the synthetic polyester fibers during a short high-temperature soldering step. The contact area can be reisolated by covering it with a layer of melting adhesive [Fig. 7(b)]. Finally, Fig. 7(c) and (d) show the textile structure contacted to the electronics according to Fig. 6(I) and (II), respectively.

First results, including the implementation of a miniaturized MP3 player system, have been demonstrated, considering manufacturability aspects in a textile environment. The system contains a central microchip module, headphones, battery, and a storage card for music data, all interconnected by conductive textiles. All electronic components are encapsulated in a robust package. The principal tasks are performed by a single versatile chip, which is capable of MP3 decoding, speech recognition, and text-to-speech conversion.
The system has been integrated in specifically designed types of clothing. Specific design aspects have been considered, such as usability, comfort, and protection against mechanical stress of the system.

In the future, the system is expandable by further modules, such as fingerprint sensor chips or wireless data transceivers. The generation of electrical power from body heat as a possible solution to the power supply problem for low-energy applications has been demonstrated in [25]. The manifold of new possible applications shows that smart clothes are a prime example of the convergence of existing technologies with new applications and markets.

\section{TeXtile-BAsed Interconnect for Signal TRANSMISSION IN WEARABLES}

Electronic systems that are integrated into clothing can serve us in a very unobtrusive and natural way. Being close 


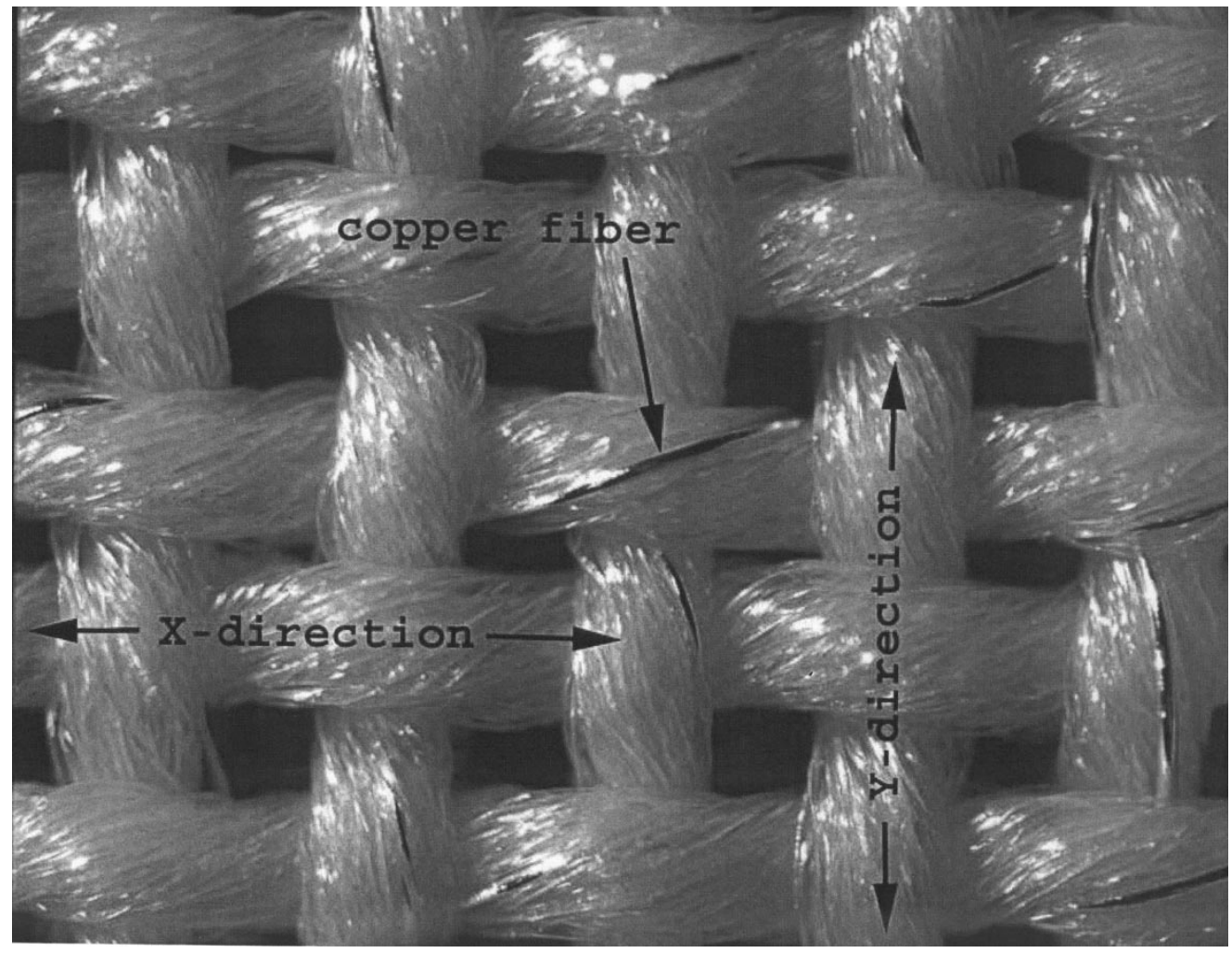

Fig. 8. Woven fabric with metal fibers.

to the body, they enable an intimate human-computer interaction. This interaction is necessary for the realization of context-aware wearable computers as described in [26]. An integration of electronics into clothing can be realized by attaching conventional electronic components to garments. However, humans prefer to wear textiles rather than "hard boxes"; thus, in conjunction with the availability of textileembedded computing, the necessity of textile-based interconnect has also emerged.

Our approach is, thus, to use conductive textiles for signal transmission. Conductive textiles already have found application in the field of electromagnetic interference (EMI) shielding and static dissipation, but there are special requirements for data transmission. We need individually addressable wires that are insulated to prevent shorts. For transmitting signals at high frequency, we need high bandwidth and sufficient signal integrity. It must be possible to process the materials using textile machinery, and the resulting materials must be robust to weaving, washing, and wearing stresses. Textiles that are used for clothing have to be made of fine and elastic fibers so that they are comfortable and lightweight. Textile technologies have been developed to manufacture fabrics out of conductive fibers that fulfill the requirements concerning processability, resistivity, and comfort. But the question still standing is whether the electrical performance is suitable for signal transmission.

To find out what range of frequencies and bandwidths textile transmission lines can be used for, we have applied methods of microwave technology. This means that wires are not only characterized by their resistance, but also by wave effects depending on the line geometries and the surrounding material. Therefore, we had to consider also the geometric structures that are created in the textile fabrication processes.

\section{A. Textiles Under Test}

For our experiments, we have used fabrics that contain insulated metal filaments. We chose conventional woven fabrics with a plain weave because this construction is the most elementary and simple textile structure. In addition, this type of material can provide a tight mesh of individually addressable wires that can be used as basic transmission lines, as well as whole circuits.

The examined fabrics contain polyester yarns that are twisted together with a copper filament. The copper filaments have a diameter of $40 \mu \mathrm{m}$ and are insulated with a polyesterimide coating. Washing and weaving tests showed that the metal fibers and the coating are extremely robust and are not damaged during typical textile handling. The critical point concerning the mechanical stability is the connection between the conductive fibers and electronic components. In the future the properties of the connections have to be investigated and optimized. This work focuses on the signal transmission characteristics.

As we wanted to find out the influence of textile geometry, we varied the yarn thickness. All fabrics have about 20 threads/cm in both directions, but the density is different according to the used polyester fineness. We manufactured 
Table 2

Examples of Geometric Variations of the Fabrics Used in the Experiments

\begin{tabular}{lll}
\hline \hline Fabric type & Dimensions & Variations \\
\hline $\begin{array}{l}\text { Fabric } 1 \\
\text { (low density) }\end{array}$ & $\begin{array}{l}\mathrm{a}=891 \mu \mathrm{m} \\
\mathrm{d}=228 \mu \mathrm{m} \\
\mathrm{a}=876 \mu \mathrm{m} \\
\begin{array}{l}\text { Fabric } 4 \\
\text { (high density) }\end{array}\end{array}$ & $\begin{array}{l}\sigma=32.9 \mu \mathrm{m} \\
\sigma=25.3 \mu \mathrm{m} \\
\sigma=25.0 \mu \mathrm{m} \\
\sigma=28.0 \mu \mathrm{m}\end{array}$ \\
Explanation: & (
\end{tabular}

fabrics with metal fibers in just one or in both directions. Fig. 8 shows a fabric with metal fibers in both directions.

Taking a closer look at the textile geometry, one can observe that fibers follow a helical path within the yarn. When the yarns are woven into a fabric, they are periodically crimped. This means that the length of the metal filament is greater than the length of the fabric. For the thinner yarn type, the metal filament is about $7.5 \%$ longer than the corresponding textile, with a tolerance of $0.5 \%$. For the thicker yarn type, where the metal fiber runs a larger helical path, this difference increases to about $25.5 \%$ with a tolerance of $2.0 \%$

$$
\begin{aligned}
& \text { Explanation: } \\
& \begin{array}{l}
d=\text { thread diameter } \\
a=\text { thread distance }
\end{array}
\end{aligned}
$$

There are several irregularities concerning the location of the fibers within the yarn as well as concerning the location of the yarns within the fabric. These variations are caused by the deformability of the textile material and the degrees of freedom in the manufacturing processes. At the level of fibers and yarns there are variations of diameters and densities (along the thread but also from thread to thread, e.g., the distance between yarns varies (as shown in Table 2). As textile material has viscoelastic behavior, inner tensions decrease over time and the geometry may change (especially in washing treatments).

\section{B. Electrical Characterization}

In order to evaluate the performance and limits of textile transmission lines, we have extracted the electrical parameters with time and frequency domain analysis and developed a theoretical model that describes the signal transmission in textiles.

1) Transmission Line Configuration: To minimize parasitic coupling at high frequencies the ground line should be close to the signal line. We decided to take metal filaments in warp direction (X-direction) as signal lines and the neighboring metal filaments on each side as ground lines. The block diagram of the measurement setup is depicted in Fig. 9. This transmission line configuration is similar to conventional coplanar waveguides (CPW) on printed wire boards.

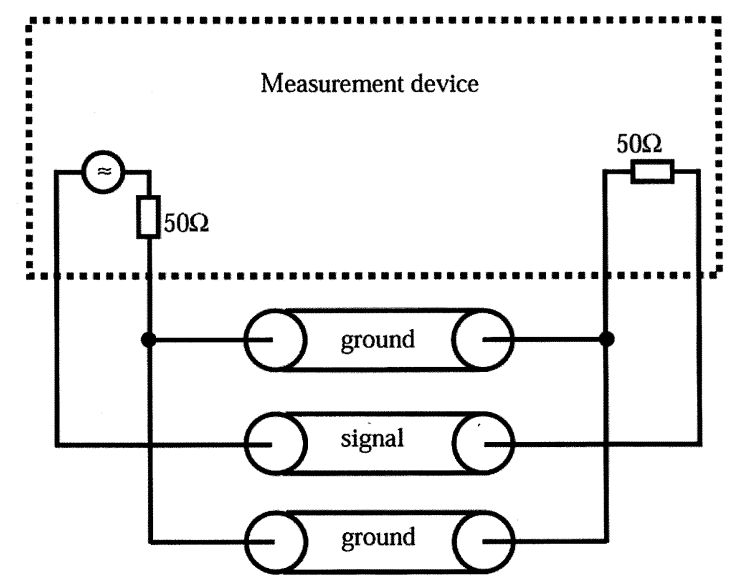

Fig. 9. Measurement setup.

Table 3

Transmission Line Configurations

\begin{tabular}{lll}
\hline \hline Configuration & $\begin{array}{l}\text { Number of } \\
\text { signal lines }\end{array}$ & $\begin{array}{l}\text { Number of } \\
\text { ground lines }\end{array}$ \\
\hline GS & 1 & 1 \\
GSG & 1 & $2(1$ on each side $)$ \\
GSSG & 2 & $2(1$ on each side $)$ \\
GSSSG & 3 & $2(1$ on each side $)$ \\
\hline \hline
\end{tabular}

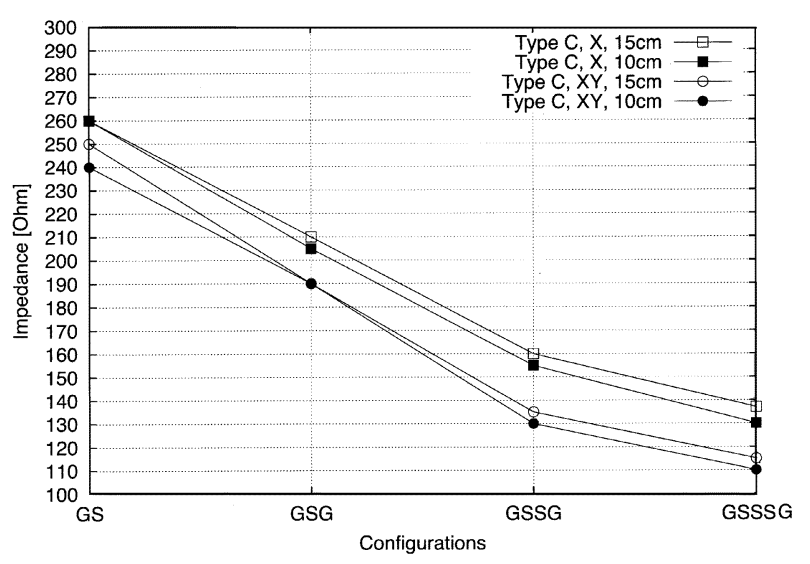

Fig. 10. Measured impedance of the different transmission line configurations.

We varied the number of signal fibers $S$ and ground fibers $G$. A list of all investigated configurations is given in Table 3.

2) Impedance Measurement: We have investigated the characteristic impedance of the textile transmission lines. As we expect the textile geometric variations to have influence on the impedance, we have measured the signal reflections along the transmission line with time domain reflectometry. Fig. 10 shows typical measured line impedances for the investigated transmission lines.

Results for the same yarn and fabric types are connected with lines to illustrate the relation between configuration and line impedance. The XY-fabrics (metal fibers in both directions) have lower impedances due to a higher capacitance that is caused by coupling effects to the floating lines in $\mathrm{Y}$ direction. The textiles with metal only in $\mathrm{X}$ direction have lower capacitance and inductance and, therefore, provide 


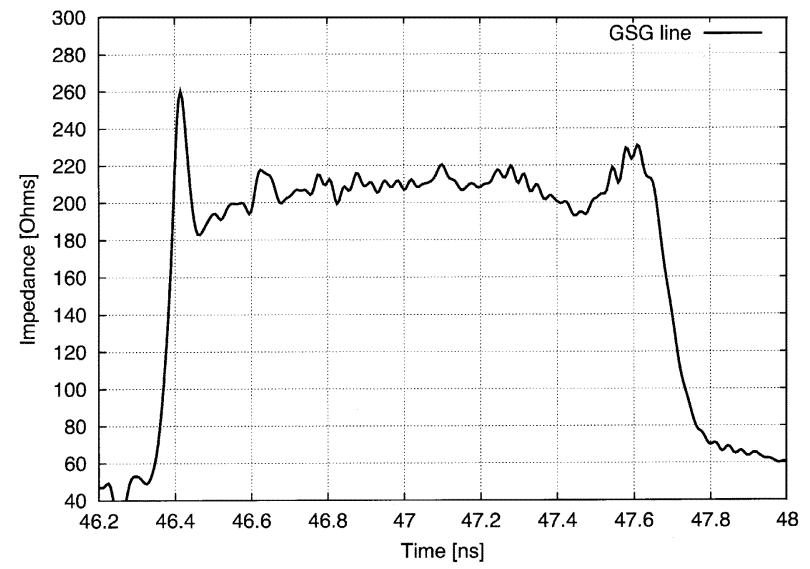

Fig. 11. Impedance profile of $15-\mathrm{cm}$ transmission line.

faster signal propagation than the textiles with metal in $\mathrm{XY}$ direction.

The results of the four configurations are comparable to coplanar waveguides on printed circuit boards (PCBs): increasing the signal line width by adding more parallel copper fibers decreases the line impedance. Using fabrics with smaller distances between the threads (that means between signal and ground lines) would enable a lower line impedance, but $50-\Omega$ lines seem difficult to achieve. The time-domain reflectometry measurements show impedance variations along the textile signal lines (see Fig. 11: the time on the horizontal axis corresponds to the position along the signal line). These variations are caused by the geometric irregularities in the fabrics. In the following, we show the effects of these variations on the predictability of the line impedance.

3) Impedance Simulation: In order to predict the impedance of different fabrics and line configurations, we modeled the textile transmission lines with a 2.5-dimensional electromagnetic field solver (Sonnet EM Suite 7.0). This way, we were able to have a better understanding of how the textile fabrication tolerances affect the line impedance. To simplify the model and reduce the computation time, the woven fabric structure was regarded as homogenous material with an equivalent dielectric permittivity determined by measurements. The metal fibers are modeled as planar metal strips between two dielectric textile layers (Fig. 12).

The simulated impedances matched well the measured results. The simulation results showed that with the given geometric variations an accuracy of $\pm 5 \%$ to $\pm 10 \%$ for the characteristic impedances is achievable. Within this tolerance, it is possible to effectively terminate a textile signal bus. With our model, different fabrics and line configurations can be simulated, so that it is possible to optimize the textile lines and to achieve defined impedance values.

4) Frequency Characterization: In order to determine the bandwidth of textile transmission lines, we investigated the frequency characteristics of textile transmission lines and measured the transmission properties with a network analyzer working at up to $6 \mathrm{GHz}$. The extracted frequency characteristics revealed that the dielectric and ohmic losses

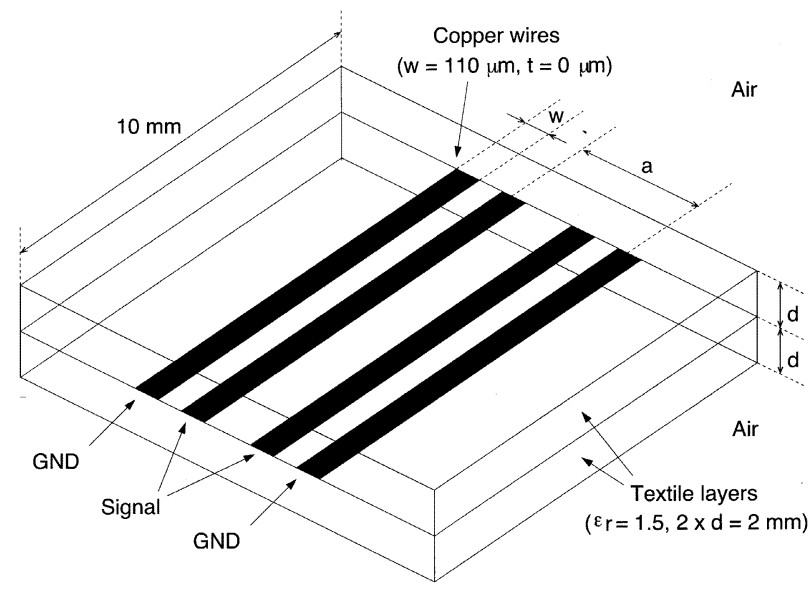

Fig. 12. Textile model for Sonnet showing a GSSG configuration.

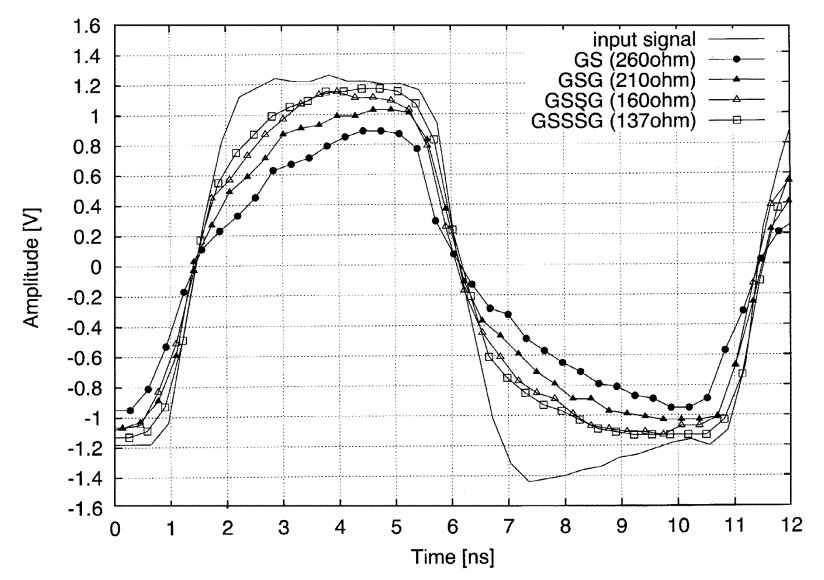

Fig. 13. 100-MHz clock signals measured through four different 20-cm-long textile transmission lines.

do not determine the line insertion loss. The loss is mainly influenced by nonuniform impedance profile along the lines and by coupling to parasitic waves in higher frequency ranges. These parasitic waves are caused by the variations of wire lengths and distances that make unequal the phases of neighboring lines. The coupling to these waves leads to loss peaks and limits the usable bandwidth. One can observe a nonmonotonic behavior of the losses and some deep minima above $1 \mathrm{GHz}$. Below $1 \mathrm{GHz}$, the coupling to parasitic waves is weak and the attenuation is less than $0.1 \mathrm{~dB} / \mathrm{cm}$. Based on these frequency characterizations, we can draw conclusions about possible line lengths, resulting losses, and usable bandwidth. For example, for a line length of $10 \mathrm{~cm}$, a usable bandwidth of $1 \mathrm{GHz}$ can be achieved.

5) Digital Signal Transmission: We tested digital signal transmission with a line length of $20 \mathrm{~cm}$ and a clock signal with a frequency of $100 \mathrm{MHz}$. Fig. 13 shows the signal integrity of different line configurations. The more signal lines we use, the better the signal integrity, but the more energy is needed for transmitting the signal. These measurements show that signal integrity is sufficient for a reliable data transmission at $100 \mathrm{MHz}$. With a simple modulation we can, therefore, achieve a data rate of $200 \mathrm{Mb} / \mathrm{s}$. This assumption does not take into account external interferences. 


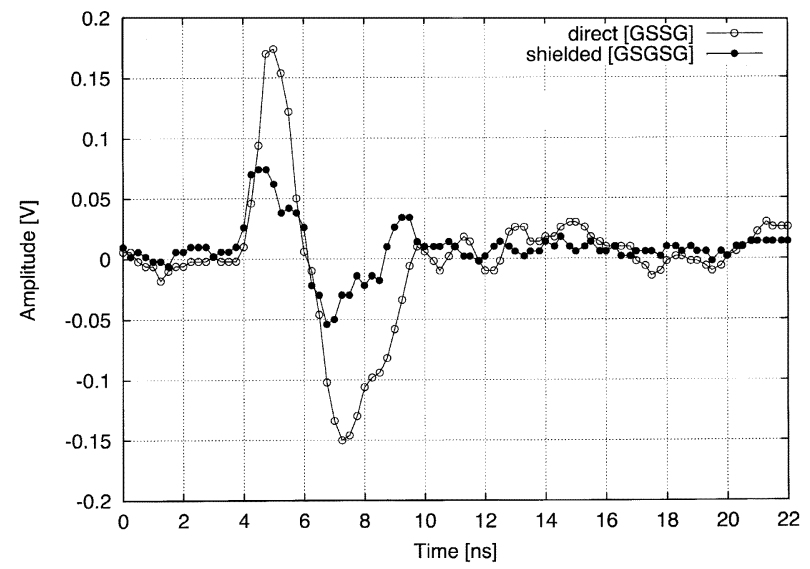

Fig. 14. Crosstalk measured on 20-cm matched load lines with and without shielding.

We expect that the most important sources of interference are neighboring signal lines that transmit other signals. So we have also investigated crosstalk effects. Fig. 14 presents the results of the far-end crosstalk for the two neighboring lines in GS and GSG configurations. The measurements were performed on two 20 -cm-long lines terminated in a matched load. The amplitude of the aggressor signal was $2.5 \mathrm{~V}$ with a rise time of $6 \mathrm{~ns}$. The ground fiber between the neighboring lines in the GSG configuration, acting as a shield, allowed reducing the crosstalk from $7.2 \%$ in GS configuration down to $2.8 \%$.

As our results show, it is possible to predict the electrical properties of different textiles and to optimize the fabrics and the signal line configurations. The final conclusion of this work is that conductive textiles provide much more than EMI shielding and power supply. Transmission lines with controlled characteristic impedance and high signal integrity up to several $100 \mathrm{MHz}$ enable new options of interconnect for e-textile-based computing.

\section{A SERVICE BACKPLANE FOR E-TEXTILE APPLICATIONS}

Given the possibility of embedding both computation and interconnect in e-textiles, the necessity of providing a set of meaningful and reasonable set of "services" for the application at hand has become important. Two broad categories of e-textile applications are envisioned, wearable, and large-scale nonwearable. Many specific applications in the field of wearable computing have been envisioned and realized, though most suffer bulky form factors [27]. In the new field of large-scale nonwearable e-textiles, applications include large-scale acoustic beamforming arrays [28], selfsteering parafoils [29], and intelligent, inflatable decoys. Both categories of e-textile applications share three common design goals: low-cost, durable, and long running.

1) Low-cost dictates the use of inexpensive, off-theshelf electronic components (COTS) and yarns as well as the design of weaves/architectures that are manufacturable in current or slightly modified textile production systems.
2) Durable dictates that the system must tolerate faults, both permanent and transient, that are inherent in the manufacture and use of the device. In addition, there is an expectation that individual components may not be repairable and that system functionality should gracefully decline as components fail.

3) Long Running dictates that the system must manage power consumption in an application-aware fashion to minimize the need for bulky batteries and/or external power recharge. Power scavenging and distributed power management are essential.

In addition to these constraints, wearable applications should have a comfortable, flexible, and unobtrusive form factor if they are to be adopted by a large number of users, while nonwearable applications should be able to operate with hundreds to thousands of components on fabrics that are tens of meters in length. By their nature, wearable e-textiles have a more complex form factor and are in motion more often than their nonwearable counterparts.

E-textiles are in a transition from a few isolated computing and sensing elements on the fabric to a network of many computing and sensing elements distributed over the entire textile. Compared to previous work in distributed systems, e-textiles will be physically spread over a relatively smaller space, but will have a greater dependence on physical locality of computation, lower bandwidth for communication, and tighter constraints on energy usage. Thus, e-textiles represent an extreme corner of the computing design space. Based on this design space, this section seeks to rationalize a set of services that form a "backplane" for a wide array of e-textile applications, including wearable and nonwearable applications.

\section{A. Application Case Studies}

This section describes two e-textile applications and identifies their common software service needs. In the category of wearable computing, a garment designed to provide the user with precise location information within a building is analyzed. A large-scale, nonwearable acoustic beamforming array is analyzed later in the section. Both textiles are woven as opposed to alternative textile manufacturing techniques such as knitting, embroidering, or nonwoven technologies. The physical media for communication on these textiles will be pliable, durable bundles of very fine metal fibers. Virtually all woven e-textiles are expected to use the new fiber batteries and solar cells under development [30], [31]. This will lead to highly distributed, redundant power supplies for e-textiles in which some parts of the textile have more remaining power than others.

These two applications were chosen as reasonably representative of the nascent e-textile technology in that each combines a range of sensors, reasonable computing requirements, and a small number of actuators. Both applications benefit from the wide distribution and large quantity of sensors that can be distributed across the textile. The mapper garment is currently under design at Virginia Tech with aspects currently in the prototyping phase. Single-cluster e-textile beamforming arrays have been constructed at Virginia Tech and a multicluster array is under construction. 
1) Mapper Garment: The mapper garment tracks the motion of the user through a structure by monitoring the user's body position, the user's movement, and the distance of the user from surrounding obstacles. Such a garment would allow users to be given directions in a building, maintenance workers to be automatically shown blueprints for their current room, or users to automatically map existing structures. The user's body position is measured by a set of piezoelectric strips woven into the clothing; by measuring the deformation along tens of strips, the physical configuration of the user's body can be detected [32]. The user's activity, such as walking upstairs, climbing a ladder, or walking on a flat surface, can be determined [32], [33]. The user's movement rate can be measured by a small set of discrete accelerometers as well as a digital compass attached to the garment. The distance from obstacles is measured using ultrasonic signals.

The primary challenge in this application is interfacing to a large number of sensors and actuators in a reliable fashion. Simply attaching the leads of every sensor/actuator to a single processing unit and power supply would not meet the design goal of a durable e-textile. In the event of a tear in the fabric, single leads running to one collection point could lead to significant rather than graceful degradation in performance. The garment needs multiple points at which analog data is converted to digital data; these conversion units, likely in the microcontroller or digital signal processor class, would need to communicate within a fault-tolerant network.

By carefully managing which sensors and processing units are active, the power requirements of the system can be reduced. For example, the computation of body position can be accomplished with varying numbers of sensors depending on the number and type of positions among which the garment is trying to distinguish [35]. By selecting sensors from around the garment according to local available power, power use can be balanced across the e-textile.

When determining the location of a wall, the garment must activate a transmitter in the direction desired and then sample a receiver for the return signal. This will accurately compute the distance, but gives no information on the direction in which it is located. To compute direction, the location of the transmitter on the body, the position of that part of the body relative to the torso, and the direction in which the user's torso is pointing must be known. A number of techniques are available for determining the location of one part of the e-textile with respect to other parts of the e-textile; in this garment, the body position is available.

2) Beamforming Array: The beamforming array textile gathers data from a large array of acoustic sensors and analyzes this data to determine the direction of an acoustic emitter (e.g., a moving vehicle or a human voice). Through the use of acoustic beamforming algorithms, a set of three acoustic sensors can identify the direction of a single emitter if the sensors and the emitter are all in the same plane. Given noise and potential miscalibrations in the acoustic data, the use of redundant acoustic sensors is advisable. As shown in Fig. 15, if the fabric is large enough, then not only the direc-

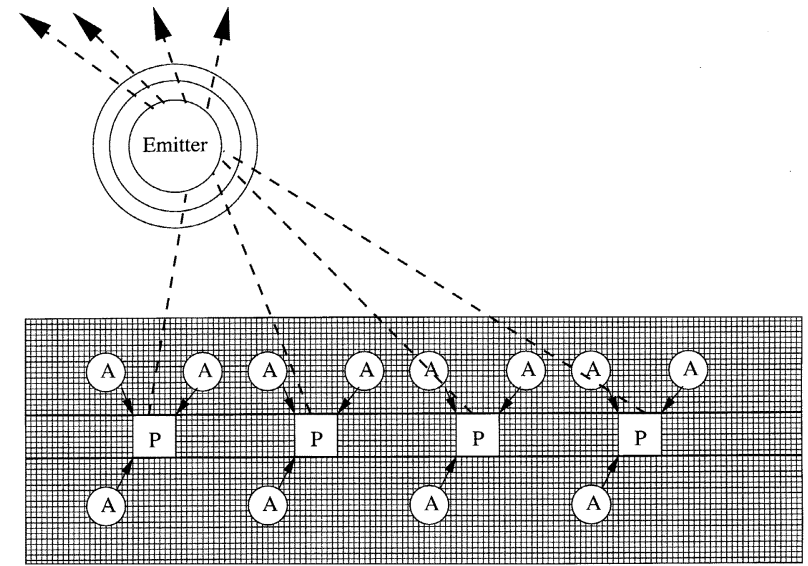

Fig. 15. The e-textile contains four processor nodes, where each processor manages a cluster of microphones. Each cluster can find the direction of the emitter; by combining the beams from each cluster, the location of the emitter can be determined. The processors in this textile are connected by a pair of communication lines.

tion, but also the location of an emitter can be found. Like the mapper garment, for reasons of robustness, the large number of sensors is not all handled by one conversion/processing node; the fabric is sprinkled with many communicating processing nodes, as shown in Fig. 15.

It is important to note that each acoustic sensor must know its location precisely with respect to the other sensors; small errors in sensor location result in increasingly larger errors as the distance to the emitter increases. Although this is not a wearable textile, it is flexible and, thus, subject to movement; at a minimum, the initial position of each acoustic sensor must be computed. In contrast to the mapper garment, the positions of each sensor must be known quite accurately. To accomplish this, the textile is augmented with speakers that are physically colocated with a subset of the acoustic sensors; by systematically activating the speakers, the distances between the microphones can be determined. Once a sufficient number of distances are known, the relative positions of all of the microphones can be computed.

The frequency, direction, and distance of a potential target all affect the optimal selection of a subset of sensors on which to perform beamforming. Because beamforming is fairly computationally demanding, it would be wasteful of resources, including power, to collect and analyze the data from the entire set of acoustic sensors. An efficient strategy, therefore, is to have a small active set of sensors look for emitters while the rest of the sensors sleep to conserve power. Upon the tentative identification of an emitter's characteristics along with an assessment of remaining power at processing nodes, an optimal set of sensors can be activated.

Once a set of sensors is selected, the time series data from those sensors must be collected at a single processor where the beamforming algorithm is to be run. If multiple beams are formed (as in Fig. 15), then the direction and intensity data must be combined at a single node; this is much less demanding of communication resources than the exchange of time series data. It is expected that some nodes along potential routes may be asleep, others out of power, and some 
broken; routes must be found in spite of these drawbacks. In addition, time series data from different nodes must be synchronized; small errors in time can lead to large errors in the computed results.

\section{B. Backplane Services Challenge}

Analysis of the application leads to the identification of the need for three types of services that are required by both applications and likely to be required by many other e-textiles:

- fault-tolerant, low-power distributed digital communication;

- computation of the physical configuration of the textile;

- selection of processing elements for tasks based on location, capability, and available energy.

Clearly, other services, such as distributed clock synchronization, were identified explicitly or implicitly in the case studies. This discussion, however, focuses on those services that have aspects that are particularly challenging in the context of e-textiles. Each of these services requires both hardware and software support. These services are described in the following sections, where the nature of the service provided is described, along with implementation issues specific to e-textiles.

1) Communication: As the case study application analysis shows, the processing elements in the textile require low-power, fault-tolerant communication. At the application level, the addressing scheme used in e-textiles for most applications should resemble schemes proposed for distributed sensor networks [36] more than it resembles TCP/IP. In the garment mapper, the natural method of addressing an ultrasonic transmitter is to address a location on the user's body, e.g., "a sensor on the left arm." In the beamforming application, the sensors are selected based on their physical location, e.g., "a time series from a sensor approximately five feet to my left."

The routing of data in an e-textile may be a completely unique networking situation. The underlying physical connections (wires) are connected in a two-dimensional (2-D) interconnection pattern that, absent faults, does not change. This 2-D structure is embedded in three-dimensional (3-D) space, and because of the flexibility of the fabric, that embedding will change over time. Thus, the route from node A to node $\mathrm{B}$ does not change, absent faults in the network. The node "five feet to your left" will change, however, as the fabric changes shape. Note that in the case of wearables, the embedding relative to the body is more or less constant; the sleeve of your shirt generally stays on your arm in the same orientation. The embedding of a nonwearable is subject to more change. Achieving "node A to node B" connectivity, and to some extent addressing parts of the body, is a relatively simple task that can rely on traditional networking approaches. Providing the application programmer with a service in 3-D space that efficiently uses the underlying connectivity is more challenging.
2) Physical Configuration Information: As shown in the previous section, it is clear that the implementation of a high-level application programming interface (API) for communication requires that the routing service know the embedding of the e-textile in three-space (or at least on the user's body). To operate effectively, e-textile applications must also have knowledge of this embedding; it is not enough to merely address messages by location. For example, the mapper garment must know the shape of the textile to infer the activity of the user; it must also know which ultrasonic sensors are in a position to sense a particular wall. Note that the first requirement is for the shape of the textile relative to itself, while the second requirement is for the shape of the textile relative to its environment.

3) Processing Element Selection: As with any efficient distributed system, a decentralized service for assigning tasks must be implemented. Two features of e-textiles push the requirements on this service into an extreme of the distributed system design space. First, the sensors and actuators available to processors on the textile each have different capabilities; even if every sensor/actuator is of the same type, they are located in different parts of the fabric and, therefore, provide different functions. By design, some level of redundancy does exist to allow the e-textile to survive faults. The second feature is the locally distributed power on e-textile systems. Unlike existing low-power systems that have a centralized power source, batteries and power scavengers are distributed across the fabric, giving each processor access to local power. Because the local power supplies will be depleted and recharged at different rates, the task assignment service must predict and balance remaining power across the textile.

The API for task assignment will provide for two basic interactions, advertising task capabilities, and advertising task needs. Applications will advertise the capability to perform application level functions such as "sense bending at the knee," "acquire a time series in the three meters northeast of center," or "move the west edge of the textile."

\section{DYNAMIC POWER AND FAULT-TOLERANCE MANAGEMENT}

To support a given set of services for power and fault-tolerance management, several possible solutions exist under given levels of redundancy (such as code migration or remote execution) [37], [38], [16]. This section investigates the benefits of a newly proposed scheme, precopying with remote execution, over a baseline code migration technique. The investigation is performed in the context of an e-textile-based system with energy and fault-tolerance constraints. The study is performed using beamforming as a driver application, as it lends itself well to partitioning on the e-textile computing substrate. However, techniques described in this section are general and can be applied to many applications involving textile-area computing arrays, such the prototypes currently developed by the COATNET research group at Carnegie Mellon University. 


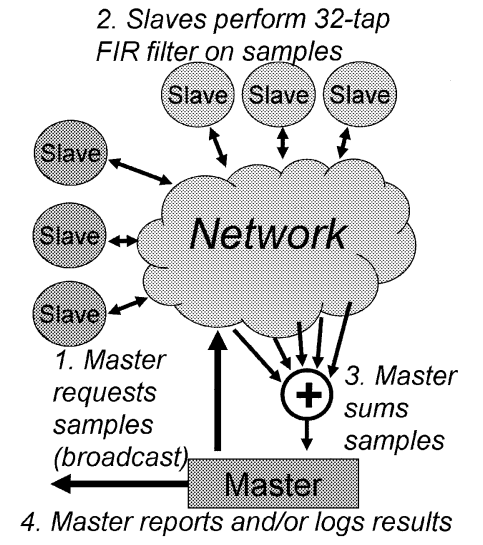

Fig. 16. Organization of beamforming application. Application is partitioned across master and slave nodes. The arrows in the figure indicate communication.

\section{A. Driver Application-Beamforming}

As a driver application, we have considered beamforming. A beamformer consists of an array of sensors working in conjunction with a centralized processor with the objective of estimating source location and content of propagating wave signals in the presence of noise. The beamforming implementation considered in this work consists of repetitive rounds in which all computational nodes participate in accomplishing the overall goal of signal recovery. At the beginning of a round, the master node sends a broadcast message to all slave nodes instructing them to begin sampling. Next, each slave obtains a sample from its attached sensor and performs a $K$-tap finite-impulse response (FIR) filter operation on this sample. It then waits for its predetermined time slot, based on its identification number, to send the result to the master node for analysis. During this analysis step, the master node combines the individual samples to obtain a composite reading for that sampling period. Finally, at the completion of a round, the master node waits for the beginning of the next round to send the next sample request broadcast and continue operation. This work focuses on the slave nodes, the master node, and the network linking together the individual slaves to the master node, as shown in Fig. 16.

\section{B. Application Remapping}

In the presence of exceptional conditions, such as critically low levels of energy resources or increasingly rampant intermittent failures, it is desirable to remap application execution from one device (or set of devices) to another. Deciding if and when to perform remapping involves tradeoffs. With low energy resources, remapping should occur early enough so as to have sufficient energy to complete. Acting too early can lead to unused energy resources going to waste, while acting too late may result in failure. One possibility for remapping the beamforming application is the use of code migration to remap slave applications executing on nodes with diminished energy resources to redundantly deployed devices with ample energy supply. With this baseline code migration, the energy resource threshold at which migration is performed must be set conservatively so as to ensure migration even in the presence of intermittent link failures. To provide more flexibility over this baseline code migration scheme, a new mechanism for performing migration is proposed herein, which permits the staging of the migration process so that effectively lower thresholds may be used which still guarantee migration.

1) Code Migration: In the ideal case, migrating an executing application will mean the application itself can be unaware of this move and can continue execution on the destination host without any change in behavior. This transparent migration requires that the entire state of the executing application (code, data, machine state, and state pertinent to any underlying system software) must be migrated faithfully. Such migration is, however, often too costly in terms of data that must be transmitted to the target host and in terms of implementation complexity.

A desirable compromise is to implement applications in a manner in which they can be asynchronously restarted while maintaining persistence for important state. Fig. 17 illustrates such a solution. The sensor node consists of a processor and memory. The memory space is partitioned between that used for the application, and memory dedicated to the device's firmware. The memory region in which the application runs is occupied by the different parts of the running application-its code (text), initialized data (data), uninitialized data (bss), stack, and heap, as illustrated by the blowup in Fig. 17. By placing information that must be persistent across restarts in the data and bss segments of the application, it is possible to maintain state across migration while only transferring the text, data, and bss segments. Each node has preloaded into it firmware referred to as the monitor. This firmware is responsible for receiving applications and loading them into memory. Each application can transmit its text, data, and bss segments, and when these are loaded by a remote monitor, the migration is complete.

Each slave node attempts to migrate its application code when the available energy left falls below a certain threshold $B_{\text {low }}$. This threshold must be chosen conservatively to ensure that each slave node can successfully migrate in the presence of bus collisions and link errors before they completely deplete their energy resource and die.

2) Precopying With Remote Execution: We propose precopying with remote execution (PCRE) as a technique to improve on code migration and remote execution, borrowing ideas from both. The primary goal is to distribute the time at which migration of application code occurs without actually transferring execution of applications until energy resources fall to a critical threshold. At this point, a single message is sent to the destination where the application code was previously precopied, and execution is transferred. This enables the threshold for migration to be set much lower, resulting in more complete usage of the energy resource. As will be shown in Section VII-D, the distribution of the migration of different nodes in time has the added benefit of reducing the number of network collisions during migration. 


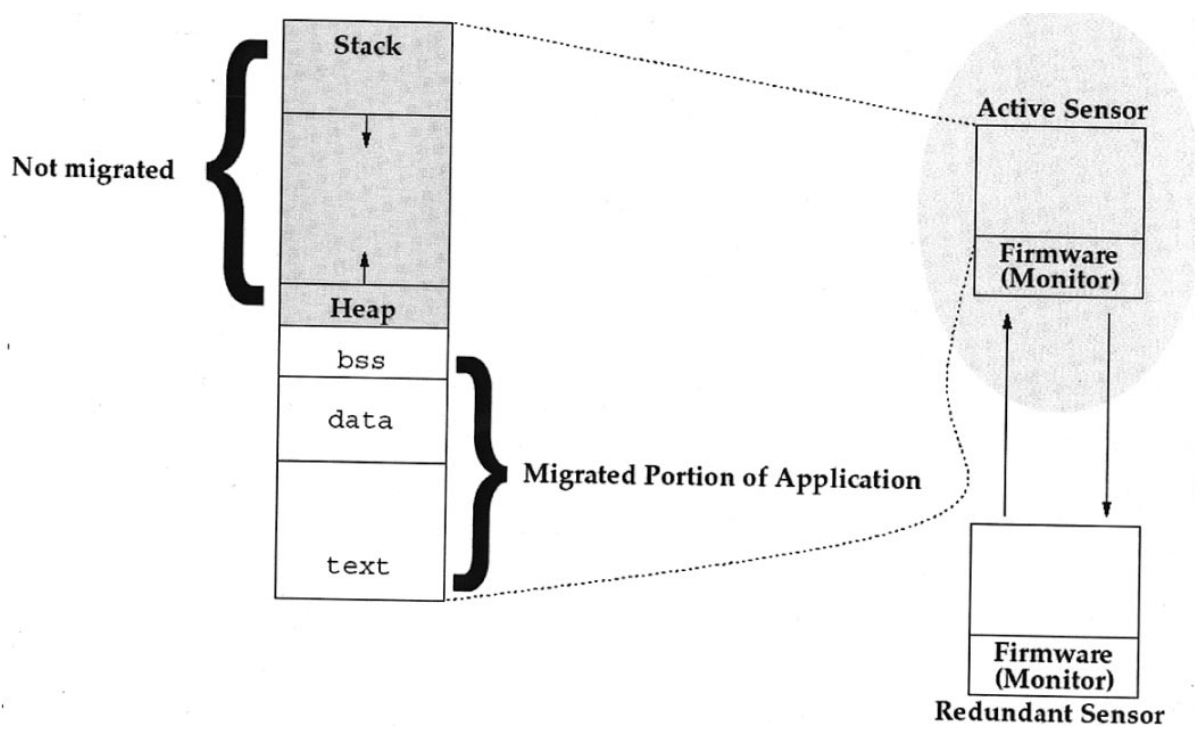

Fig. 17. Lightweight migration of application code and state. Only program code, data, and uninitialized data are transferred during migration.

Table 4

Simulation Variables and Their Associated Values

\begin{tabular}{ll}
\hline \hline Simulation Parameter & Value \\
\hline Operating Modes ( each node ) & $60 \mathrm{Mhz} @ 3.3 \mathrm{~V}$ \\
& $20 \mathrm{Mhz} @ 1.1 \mathrm{~V}$ \\
Battery Size ( each node ) & $1 \mathrm{mAh}$ \\
Trans. and Rec. Power & $100 \mathrm{~mW}$ \\
Link Speed & $200 \mathrm{kbps}$ \\
Frame Size & $1024 \mathrm{bits}$ \\
Frame Headers & $288 \mathrm{bits}$ \\
Node Failure Probability & $1 \mathrm{E}-8$ \\
Correlation Coefficient & 0.1 \\
Baseline Migration Threshold $\left(\mathrm{B}_{\text {low }}\right)$ & 0.6 \\
PCRE Migration Threshold $\left(\mathrm{B}_{\text {vlow }}\right)$ & 0.06 \\
\hline \hline
\end{tabular}

\section{Experimental Setup}

The simulator used in this study is built around a publicly available energy estimating architectural simulator for the Hitachi SuperH embedded processor architecture. It models a network of embedded processors, each consisting of a Hitachi SH3 microcontroller, memory, and communication interface. The simulation environment permits the instantiation of a large number of nodes as well as interconnection links (it has been used to simulate networks on the order of hundreds of nodes) and attached batteries. Failures are modeled in the links that interconnect the processing elements in the fabric. These failures are modeled after intermittent electrical failures, with an adjustable probability of failure (referred to as the failure rate in Section IV). These probabilities of failure are with respect to one simulation time step. For example, a stated failure rate of $1 \mathrm{E}-8$ implies a probability of failure of $1 \mathrm{E}-8$ per simulation step. The correlation coefficient is the likelihood that a link error will cause a node error on a node connected to that link.

1) Simulation Setup: Several relevant simulation variables and their associated values assigned for these experiments are given in Table 4. All experiments employ a beamforming implementation with one master node and ten slave nodes. In every experiment, half the slave nodes are inactive and used as targets for migration. As can be seen from Table 4, each slave node is attached to a battery with a capacity of $1.0 \mathrm{mAh}$. For the design alternative of using a larger battery per slave node, each slave node is attached to a battery with twice the capacity, or $2.0 \mathrm{mAh}$. These battery capacities are on the order of ten times smaller than a common wristwatch battery. Such a small battery in today's technology would have a size of $1 \mathrm{~mm}^{2}$ and cost much less than a dollar. This size of battery was chosen to limit the simulated system's lifetime and make simulation possible in a reasonable amount of real time. The master node's battery capacity is large enough to guarantee that it continues functioning throughout the simulation. The threshold for PCRE ( $B_{\text {vlow }}$ in Table 4 ) is ten times lower than that for baseline migration $B_{\text {low }}$ because only one frame needs to be transmitted to complete migration. Eleven experiments are conducted to compare PCRE with two other design solutions: using the baseline migration scheme as defined in Section VII-B, and using a larger battery per processing node instead of using code migration at all. The details of each configured experiment are shown in Table 5.

Two topologies for interconnecting the master, slave and redundant nodes are considered, and are depicted in Fig. 18. In one topology, referred to as Dual-Bus, shared communication buses are employed, one for exchanging samples between the master and slave nodes and the other for migration of slaves to redundant nodes. In the second topology, the single shared bus for the exchange of samples is maintained, but Dedicated Migration Links are employed for migrating the slaves to the redundant nodes. In Fig. 18, the redundant nodes are the darker shaded nodes at the bottom, and the slave nodes are the lighter shaded nodes at the top. To investigate the robustness of the new code migration technique in the presence of faults, simulations 
Table 5

Details Showing How Each Experiment Was Set Up

(L.E. $=$ Link Errors, N.E. = Node Errors, Ind. = Independent, Cor. $=$ Correlated)

\begin{tabular}{cccc}
\hline \hline $\begin{array}{c}\text { Exp. } \\
\#\end{array}$ & Topology & Errors? & $\begin{array}{c}\text { CPU } \\
\text { Speed }\end{array}$ \\
\hline 1 & Dual-Bus & None & $60 \mathrm{Mhz}$ \\
2 & Dual-Bus & L.E. & $60 \mathrm{Mhz}$ \\
3 & Dual-Bus & N.E. & $60 \mathrm{Mhz}$ \\
4 & Dual-Bus & Ind. L.E. + & $60 \mathrm{Mhz}$ \\
& N.E. & \\
5 & Dual-Bus & Cor. L.E. + & $60 \mathrm{Mhz}$ \\
& & N.E. & \\
6 & Dedicated Migration Link & None & $60 \mathrm{Mhz}$ \\
7 & Dedicated Migration Link & L.E. & $60 \mathrm{Mhz}$ \\
8 & Dedicated Migration Link & N.E. & $60 \mathrm{Mhz}$ \\
9 & Dedicated Migration Link & Ind. L.E. + & $60 \mathrm{Mhz}$ \\
& & N.E. & \\
10 & Dedicated Migration Link & Cor. L.E. + & $60 \mathrm{Mhz}$ \\
& & N.E. & \\
11 & Dual-Bus & None & $60 \mathrm{Mhz}$ \\
\hline \hline
\end{tabular}

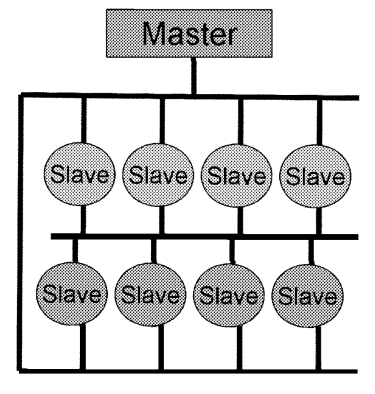

Dual-Bus

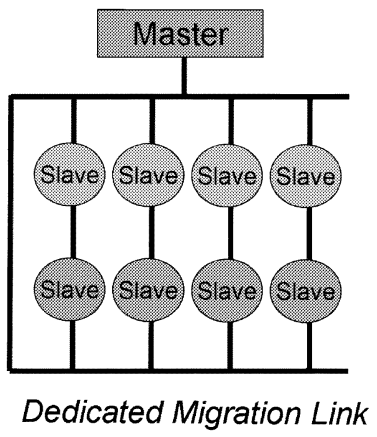

Dedicated Migration Link
Fig. 18. Two topologies considered in experiments: Dual Bus and Dedicated Migration Link topologies.

are performed with intermittent failures in the slave and redundant nodes, failures in the links, as well as independent and correlated failures in both the nodes and the links. Table 4 shows the node and link failure probabilities per simulation cycle. A $1 \mathrm{E}-8$ per 16.6 -ns cycle time error probability, for a link operating at $200 \mathrm{~kb} / \mathrm{s}$, translates to an error rate of $3.125 \mathrm{E}-6$ per bit transmitted.

\section{Results and Discussion}

Fig. 19 shows the number of samples received by the master node as well as the remaining usable battery energy as the application evolves for Experiment 1. In the case of PCRE, the solid line of Fig. 19 shows that the first $10 \mathrm{~s}$ is the time in which precopying is taking place by each active node, and this unique "sawtooth" shape helps to prevent possible link collisions during precopying. At approximately $35 \mathrm{~s}$, all nodes send their final frame to the redundant node to complete migration. For the baseline migration implementation, as is apparent in the solid line of Fig. 19, nodes begin migration around $15 \mathrm{~s}$ and completely resume normal operation by $20 \mathrm{~s}$. The dotted lines show that, for the baseline migration scheme, the amount of usable battery energy decreases considerably in the region of 15 to
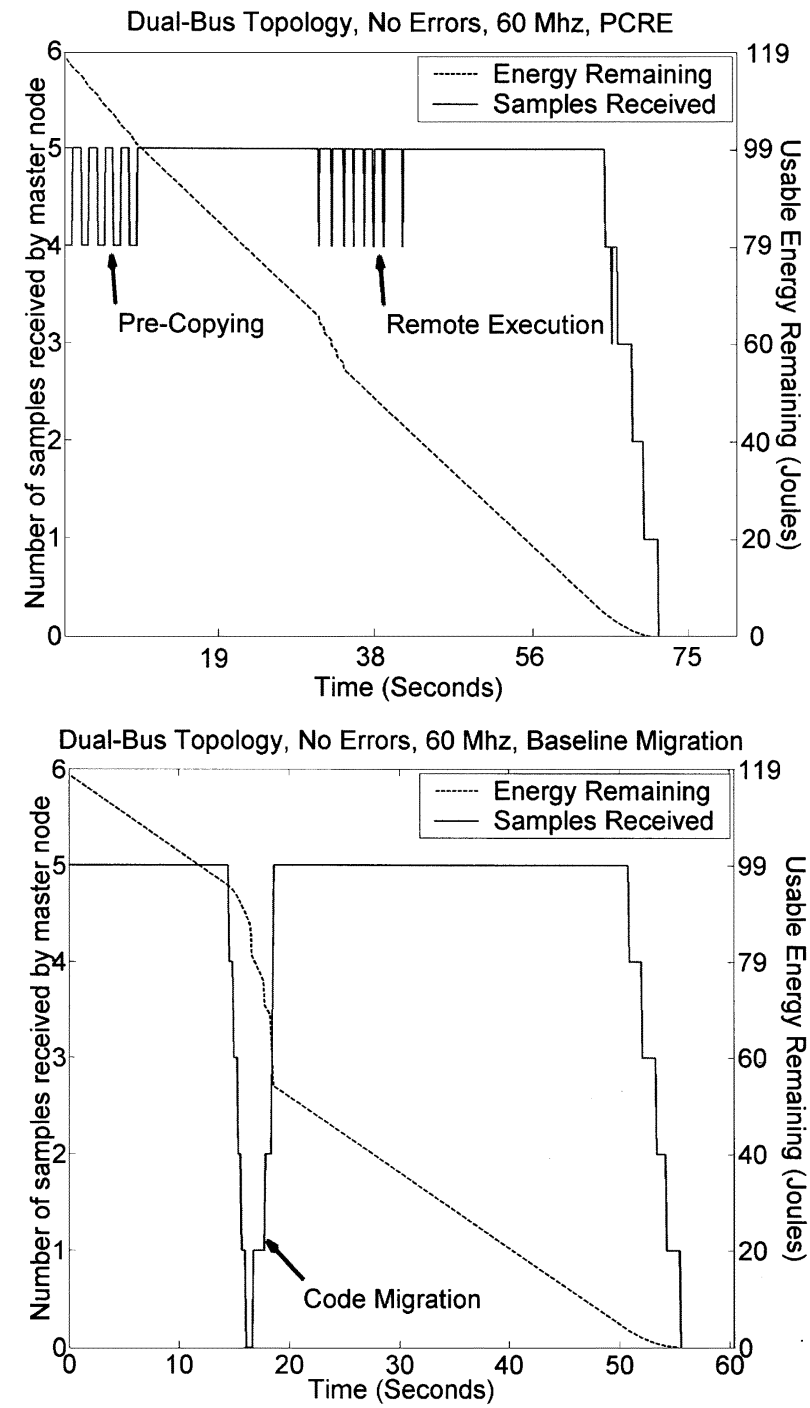

Fig. 19. Experiment 1-Dual Bus topology, no errors, 60-MHz processors.

$20 \mathrm{~s}$ because, in this region, each slave node is abandoning what remaining energy it may have left by performing the migration. The amount of usable energy remaining using PCRE also drops during the final migration stage, but not as drastically as in the baseline migration case. Summarizing results for all the experiments are included in Figs. 19 and 20. On average, PCRE increases the system lifetime over the baseline migration scheme by $57.9 \%$. For baseline migration, Experiment 9 did not successfully migrate mainly due to random node and link errors, so this impressive increase is largely due to the great improvement in lifetime for this experiment (see Fig. 19). Removing this experiment from consideration results in an average system lifetime improvement of $28.6 \%$ for PCRE compared to baseline migration. Fig. 20 shows these lifetimes, normalized to the system lifetime of the "ideal" configuration where each node has twice the battery capacity and no migration occurs. Fig. 20 shows an improvement in system lifetime, the first metric on the metrics list at the beginning of this section. As it can be seen, PCRE achieves, in most cases, a system 


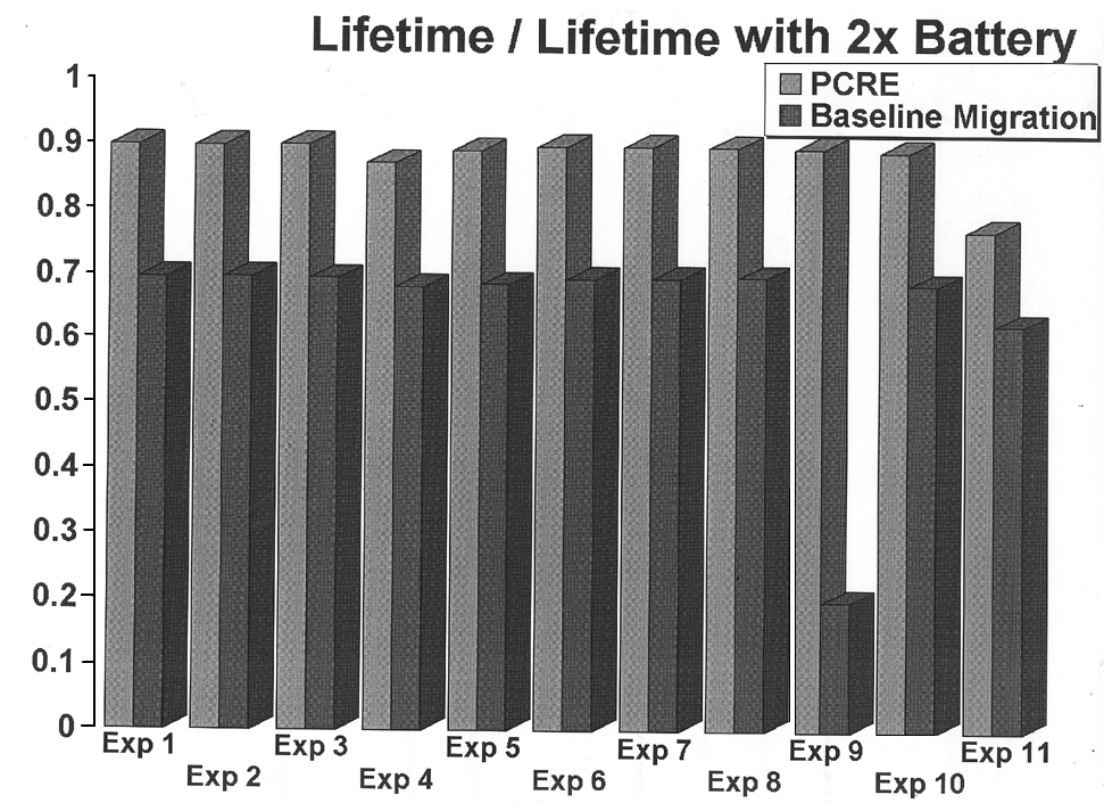

Fig. 20. System lifetime for two migration schemes, normalized to the system lifetime resulting by simply using batteries with twice the capacity.

lifetime within $10 \%$ of the ideal case while baseline migration is usually $30 \%$ worse than the ideal system lifetime. For the second metric, an improvement in the performance of the beamformer using PCRE was observed. For example, in Experiment 1, PCRE had an average of 4.698 samples received during its lifetime per round, with a standard deviation of 0.795 . Baseline migration, meanwhile, suffered somewhat, with only 4.498 samples received per round, with a higher standard deviation at 1.273. Similar arguments can be made for the other experiments, as well. Next, the energy efficiency is also improved using PCRE as opposed to baseline migration. For Experiment 1, PCRE consumes 115.26 J of its 118.8-J total capacity with a system lifetime of $72.3 \mathrm{~s}$, and baseline migration consumes $95.2 \mathrm{~J}$ of its $118.8-\mathrm{J}$ total capacity with a system lifetime of $54.4 \mathrm{~s}$. This gives PCRE a ratio of 0.627 -s lifetime per joule consumed, and the baseline migration 0.571-s lifetime per joule consumed. Therefore, for Experiment 1, PCRE enjoyed an energy efficiency improvement of $9.8 \%$ compared to the baseline migration scheme. The final metric of interest is the average migration cost of an active node. The average migration cost analysis for each experiment is given in Fig. 21. As can be seen in Fig. 21, the migration cost is much higher for baseline migration than for PCRE in the experiments where a shared migration bus is employed. Many of the active nodes experience collisions when attempting to migrate over the shared bus, and this attributes to the increased migration cost for those experiments.

Considering the two bar graphs of Fig. 21 together, it is clear that systems with a higher network collision rate suffer with higher average migration costs. The slight increase in migration costs for Experiments 6-11 is an implementation detail of the beamforming application, and does not reflect on the PCRE approach itself.
The results of our study show that, in general, classic techniques like code migration and remote execution provide reasonable solutions for application remapping in the presence of failures or finite energy resources. Based on our experimental results, we conclude that PCRE provides the right amount of support for dynamic management of fault tolerance and power consumption by reducing the overall beamforming lifetime by $28.6 \%$ on average, as well as improving energy efficiency and beamforming performance compared to a baseline code migration technique.

\section{E-TEXTILES: LOOKING IN THE CRYSTAL BALL}

The various research efforts highlighted in Sections IV-VII demonstrate the potential for cutting edge research in the area of e-textiles. Looking ahead, the following key questions are possible venues that could be explored by researchers in the future.

- What will be the underlying computing substrate for e-textiles: computing offline, onto, or into the fabric?

- What will be the solutions for dealing with limited and finite energy resources in conjunction with faulty communication and computation?

- What will next-generation e-textiles "compute" or how they will "communicate"?

- Is it possible to achieve human performance enhancement with e-textiles?

Addressing the first question, while it is generally believed that (at least in the beginning) computation will still be done in silicon, there exists the (albeit remote) possibility of having computation really embedded into fibers [39]. Since for the next five to ten years, computation will most likely be done offline (that is, on a separate system, connected to the fabric itself) or onto the fabric (on controllers attached onto the 


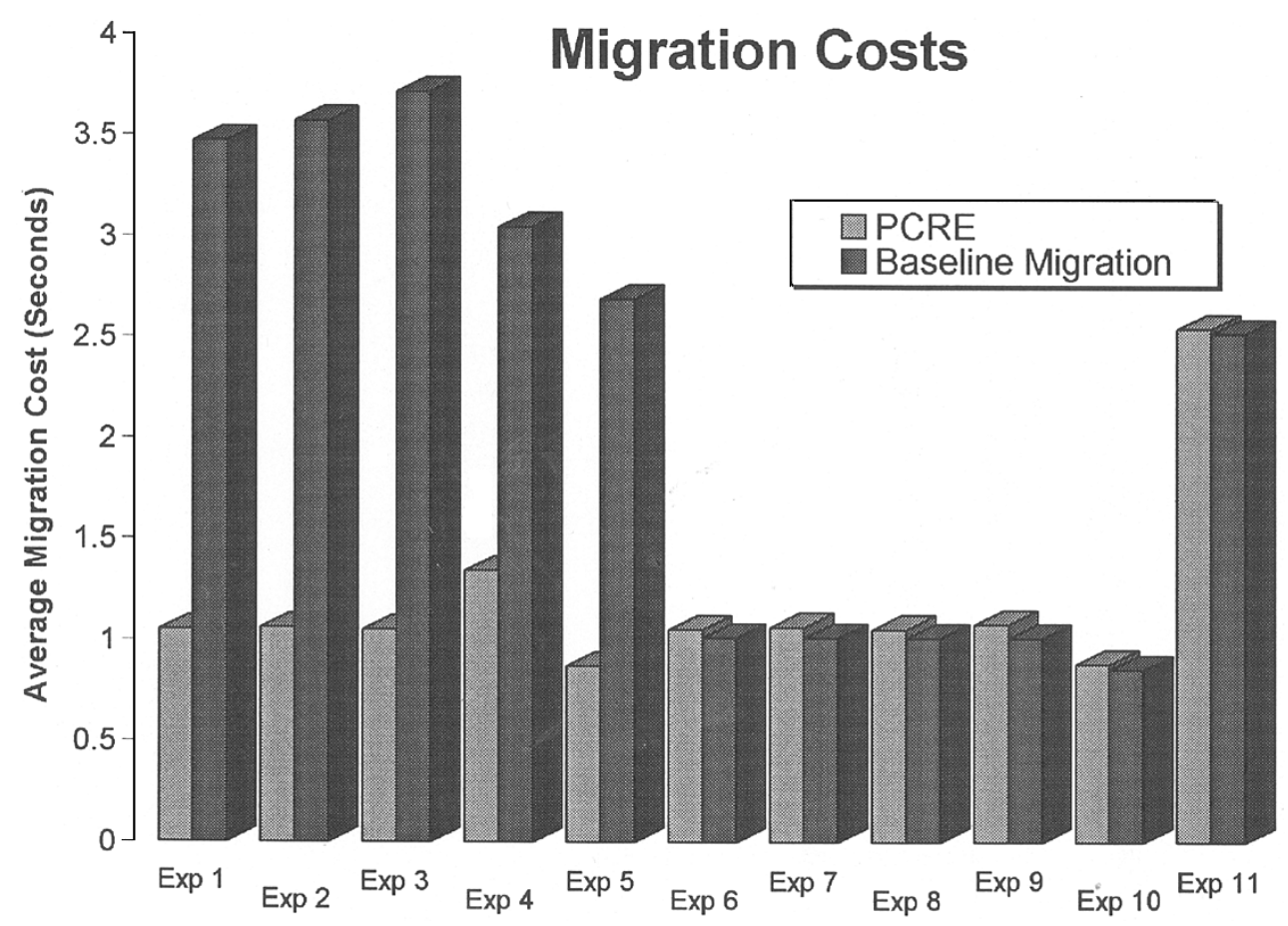

(a)

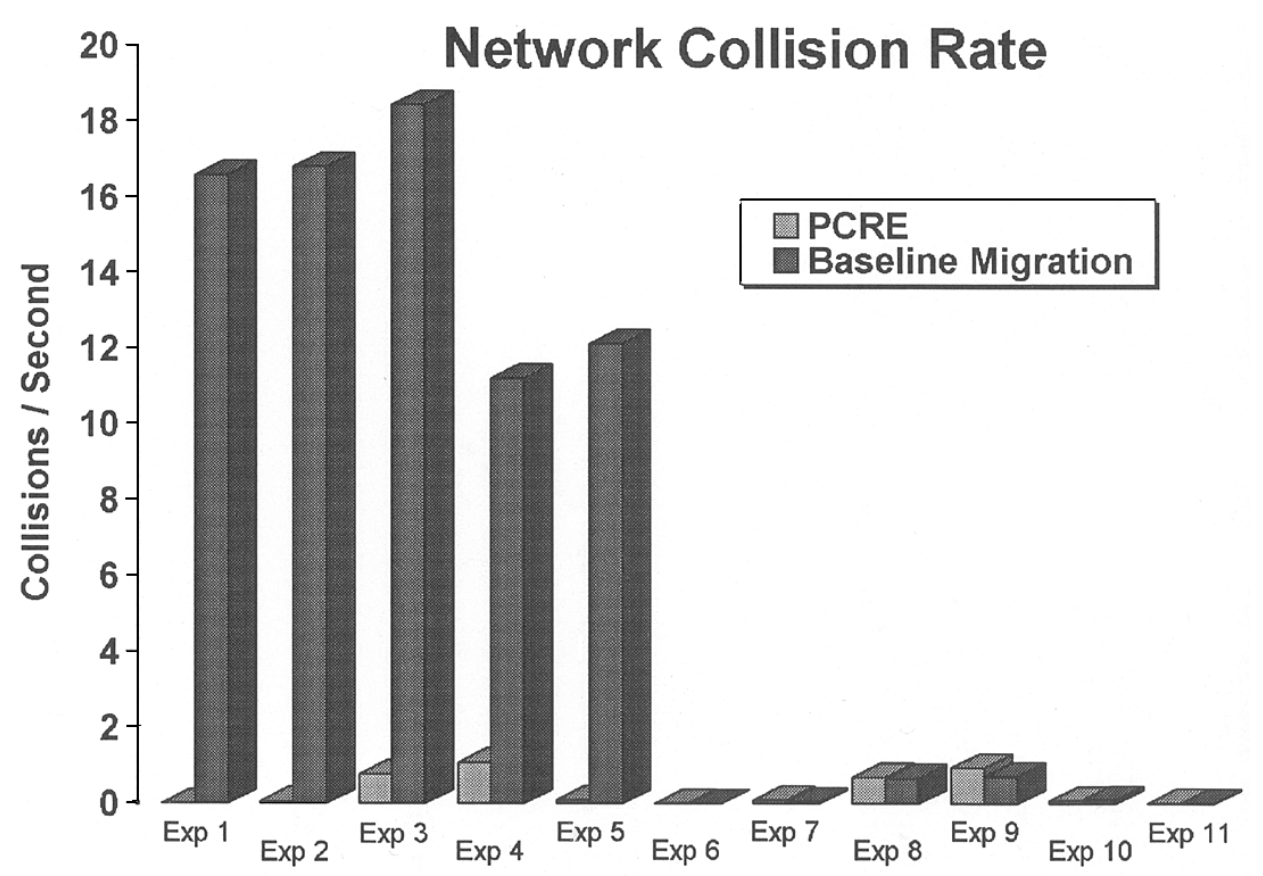

(b)

Fig. 21. Average costs of migration (time it takes to migrate code from one slave to a redundant node) and average number of collisions per second for each experimental setup.

fabric), the underlying substrate will most likely be silicon. It may be possible that, with the advent of alternative nonsilicon technologies (nano, molecular, polymer based, etc.), in the next two decades we may be able to see computation embedded seamlessly into the fabric. Nonsilicon substratebased yarns could be woven just like regular fibers, providing inconspicuous computing power for a variety of applications. What is lacking in either case, however, is a set of tools and methodologies that will be able to provide for e-textiles what SPICE has provided for integrated circuits. Irrespective of the underlying computing substrate, this research area is still in its infancy, without much support from existing tools 
and methodologies, the most important reason for which is the different model of computation and lifetime cycle (as described in Section III).

While computation is still likely to be done in silicon, and not into the yarns, the same may not be true for energy resources. Distributed batteries, possibly fuel cells or filament-type batteries woven into the fabric have already been demonstrated [40]. Since reliable operation in the presence of harsh or regular wear-and-tear environments must be ensured, possible solutions include providing added redundancy for both computation and interconnect, as well as energy sources. On the other hand, if redundancy may prove to be too expensive by requiring extra devices, a possible solution is to make sure that the interconnect and computation are designed fault free, thereby moving some of the costs from the extra devices to the manufacturing and testing costs. A common belief, though, is the fact that energy scavenging and conservation is and will be of prime importance, in conjunction with providing dynamic support for reconfiguration and remapping.

The last two questions involve the type of applications that are seen as potential candidates for mapping onto e-textiles. When thinking about context awareness or enhancing human performance awareness, e-textiles may just be the computing substrate needed for achieving it. E-textiles may prove to be the next successors of smart cards in allowing people to seamlessly move from one ambient to the other, with the clear advantage of being close to the human body and, thus, being able to provide biometric authentication, inconspicuously and without intrusion. At the same time, e-textiles will be able to provide possibly the best support to impaired individuals by enabling improved sensing, in addition to actuation, both embedded in regular garments. Finally, special segments of the market (such as medical, military, law enforcement, as well as sports training) may also benefit from the already available e-textile-based technology for vital signs monitoring or remote triage.

\section{ACKNOWLEDGMENT}

The authors would like to thank Dr. E. Lind of the U.S. Department of the Navy, Mr. D. O'Brien of the U.S. Defense Logistics Agency, and Dr. R. Satava of the Defense Advanced Research Projects Agency for identifying the need for a soldier protection system. The authors would also like to thank Dr. K. Mackenzie for his contributions to the demonstration of the "in-fabric" network at Georgia Tech, Atlanta. The authors would also like to thank the following groups, companies, and institutions for their advice and contributions: Infineon Speech Interface Group, Ingentix $\mathrm{GmbH}$, Materials and Manufacturing Group of Siemens Corporate Technology, Degussa AG, Institut für Textil- und Verfahrenstechnik Denkendorf, EDISEN-electronic, Ionity AG, Elektro-Feindraht AG, and the Deutsche Meisterschule für Mode, Munich. The authors would also like to thank J. Bonan, A. Kuhn, and I. Ruiz for their valuable help in the textile measurements.

\section{REFERENCES}

[1] S. Jayaraman, "Designing a textile curriculum for the '90s: A rewarding challenge," J. Textile Inst., vol. 81, no. 2, pp. 185-194, 1990.

[2] C. Gopalsamy, S. Park, R. Rajamanickam, and S. Jayaraman, "The wearable motherboard: The first generation of adaptive and responsive textile structures (ARTS) for medical applications," J. Virtual Reality, vol. 4, pp. 152-168, 1999.

[3] S. Park and S. Jayaraman, "Adaptive and responsive textile structures," in Smart Fibers, Fabrics and Clothing: Fundamentals and Applications, X. Tao, Ed, Cambridge, U.K.: Woodhead, 2001, pp. 226-245.

[4] The Georgia Tech wearable motherboard: The intelligent garment for the 21st century (1998). [Online]. Available: http://www.smartshirt.gatech.edu

[5] R. Rajamanickam, S. Park, and S. Jayaraman, "A structured methodology for the design and development of textile structures in a concurrent engineering environment," J. Textile Inst., vol. 89, no. 3, pp. 44-62, 1998.

[6] S. Park, C. Gopalsamy, R. Rajamanickam, and S. Jayaraman, "The wearable motherboard: An information infrastructure or sensate liner for medical applications," in Studies in Health Technology and Informatics. Amsterdam, The Netherlands: IOS Press, 1999, vol. 62 , pp. 252-258.

[7] S. Park and S. Jayaraman, "Quality of life in the internet age: Role of the Georgia Tech smart shirt," Atlanta Med., vol. 74, no. 4, pp. 24-28, Winter 2001.

[8] K. Mackenzie, D. Hudson, S. Maule, S. Park, and S. Jayaraman, “A prototype network embedded in textile fabric," in Proc. Int. Conf. Compilers, Architecture, and Synthesis Embedded Systems, 2001, pp. 188-194.

[9] S. Park, K. Mackenzie, and S. Jayaraman, "The wearable motherboard: A framework for personalized mobile information processing (PMIP)," presented at the ACM/IEEE 39th Design Automation Conf., New Orleans, LA, 2002.

[10] S. Park and S. Jayaraman, "E-textiles," Ind. Fabric Prod. Rev., vol. 87, no. 11, pp. 64-75, Oct. 2002.

[11] E. R. Post and M. Orth, "Smart fabric, or wearable clothing," in Proc. Int. Symp. Wearable Computers, 1997, pp. 167-168.

[12] E. R. Post, M. Orth, P. R. Russo, and N. Gershenfeld, "E-broidery: Design and fabrication of textile-based computing," IBM Syst. J., vol. 39, no. 3-4, pp. 840-860, 2000.

[13] M. Gorlick, "Electric suspenders: A fabric power bus and data network for wearable digital devices," in Dig. Papers Int. Symp. Wearable Computers, 1999, pp. 114-121.

[14] J. Rantanen, T. Karinsalo, M. Mkinen, P. Talvenmaa, M. Tasanen, and J. Vanhala, "Smart clothing for the arctic environment," in Proc. Int. Symp. Wearable Computers, 2000, pp. 15-23.

[15] R. Marculescu and D. Marculescu, "Does $Q=M C^{2}$ ? On the relationship between quality in electronic design and the model of colloidal computing," in Proc. Int. Symp. Quality Electronic Design, 2002, pp. 451-457.

[16] D. Marculescu, R. Marculescu, and P. Khosla, "Challenges and opportunities in electronic textiles modeling and optimization," in Proc. ACM/IEEE 39th Design Automation Conf., 2002, pp. 175-180.

[17] _ "Challenges and opportunities in electronic textiles modeling, analysis and optimization," presented at the Int. Interactive Textiles for the Warrior Conf., Boston, MA, 2002.

[18] S. Ditlea, "The PC goes ready to wear," IEEE Spectr., vol. 37, pp. 35-39, Oct. 2000.

[19] D. Eves, J. Green, C. van Heerden, J. Mama, and S. Marzano, New Nomads-An Exploration of Wearable Electronics. Rotterdam, The Netherlands: Uitgeverij 010, 2001.

[20] W. D. Hartmann, K. Steilmann, and A. Ullsperger, High-Tech Fashion. Witten, Germany: Heimdall Verlag, 2000.

[21] P. Lukowicz and G. Tröster, "Packaging issues in wearable computing," in Proc. CPD 2000, pp. 19-22.

[22] Infineon Technologies_-products and product categories [Online]. Available: http://www.infineon.com/products

[23] S. Jung, C. Lauterbach, and W. Weber, "A digital music player tailored for smart textiles: First results," presented at the Avantex Symp., Frankfurt, Germany, 2002.

[24] B. Burchard, S. Jung, A. Ullsperger, and W. D. Hartmann, "Devices, software, their applications and requirements for wearable electronics," in Proc. ICCE, 2001, pp. 224-225. 
[25] C. Lauterbach, M. Strasser, S. Jung, and W. Weber, "Smart clothes self-powered by body heat," presented at the Avantex Symp., Frankfurt, Germany, 2002.

[26] P. Lukowicz, H. Junker, M. Stäger, T. von Büren, and G. Tröster, "WearNET: A distributed multi-sensor system for context aware wearables," in Proc. 4th Int. Conf. Ubiquitous Computing, 2002, pp. 361-370.

[27] F. Gemperle, C. Kasabach, J. Stivoric, M. Bauer, and R. Martin, "Design for wearability," in Proc. 2nd Int. Symp. Wearable Computers, 1998, pp. 116-122.

[28] R. Parker, R. Riley, M. Jones, D. Leo, L. Beex, and T. Milson, "STRETCH-An E-textile for large-scale sensor systems," presented at the Int. Interactive Textiles for the Warrior Conf., Cambridge, MA, 2002.

[29] E. Urban, "Parafoils and acoustic arrays," presented at the Int. Interactive Textiles for the Warrior Conf., Cambridge, MA, 2002.

[30] B. C. Kim, G. M. Spinks, R. John, and G. G. Wallace, "Electroformation of conducting polymers in a hydrogel support matrix," in Proc. Polymer 2000, vol. 41, pp. 1783-1790.

[31] L. Samuelson, F. Bruno, J. Kumar, R. Gaudiana, and P. Wormser, "Conformal solar cells for the soldier," presented at the Int. Interactive Textiles for the Warrior Conf., Cambridge, MA, 2002.

[32] J. Edmison, M. Jones, Z. Nakad, and T. Martin, "Using piezoelectric materials for wearable electronic textiles," in Proc. 6th Int. Symp. Wearable Computers, 2002, pp. 41-48.

[33] A. R. Golding and N. Lesh, "Indoor navigation using a diverse set of cheap, wearable sensors," in Proc. 3rd Int. Symp. Wearable Computers, 1999, pp. 29-36.

[34] K. Van Laerhoven and O. Cakmakci, "What shall we teach our pants?," in Proc. 4th Int. Symp. Wearable Computers, 2000, pp. 77-83.

[35] K. Van Laerhoven, A. Schmidt, and H. Gellersen, "Multi-sensor context aware clothing," in Proc. 6th Int. Symp. Wearable Computers, 2002, pp. 49-56.

[36] C. Intanagonwiwat, R. Govindan, and D. Estrin, "Directed diffusion: A scalable and robust communication paradigm for sensor networks," presented at the 6th Annu. Int. Conf. Mobile Computing and Networks (MobiCOM 2000), Boston, MA, 2000.

[37] D. Milojicic, W. LaForge, and D. Chauhan, "Mobile objects and agents," in Proc. USENIX Conf. Object-Oriented Technologies and Systems, 1998, pp. 1-14.

[38] A. Rudenko, P. Reiher, G. J. Popek, and G. H. Kuenning, "The remote processing framework for portable computer power saving," in Proc. ACM Symp. Applied Computing, 1999, pp. 365-372.

[39] B. Gnade, T. Akinwande, G. Parsons, S. Wagner, and R. Shashidhar, "Active devices on fiber: The building blocks for electronic textiles," presented at the Int. Interactive Textiles for the Warrior Conf., Cambridge, MA, 2002.

[40] L. A. Samuelson, F. F. Bruno, J. Kumar, R. A. Gaudiana, and P. M. Wormser, "Conformal solar cells for the soldier," presented at the Int. Interactive Textiles for the Warrior Conf., Cambridge, MA, 2002.

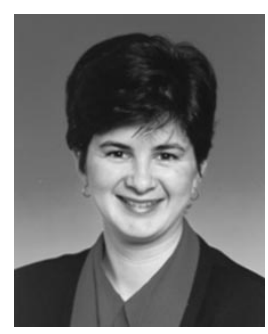

Diana Marculescu (Member, IEEE) received the M.S. degree in computer science from Politehnica University, Bucharest, Romania, in 1991 and the Ph.D. degree in computer engineering from the University of Southern California, Los Angeles, in 1998.

She is currently an Assistant Professor of electrical and computer engineering at Carnegie Mellon University, Pittsburgh, PA. Her research interests include energy aware computing, CAD tools for low power systems and emerging technologies (such as electronic textiles or ambient intelligent systems).

Dr. Marculescu is a Member of the Executive Board of the Association for Computing Machinery Special Interest Group on Design Automation (SIGDA). She is the recipient of a National Science Foundation Faculty Early Career Development Award (2000-2004) and of an ACM-SIGDA Technical Leadership Award (2003).

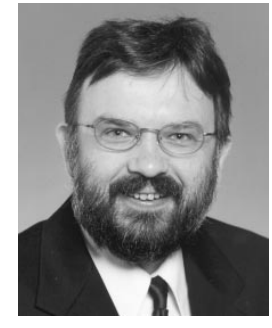

Radu Marculescu (Member, IEEE) received the $\mathrm{Ph} . \mathrm{D}$. degree in electrical engineering from the University of Southern California, Los Angeles, in 1998.

$\mathrm{He}$ is currently an Assistant Professor in the Department of Electrical and Computer Engineering at Carnegie-Mellon University, Pittsburgh, PA. His research interests include system-level design methodologies with emphasis on low-power issues, networks-on-chip, and ambient intelligence.

Dr. Marculescu has received best paper awards at the 2001 and 2003 Design and Test Conference in Europe (DATE) and the 2003 Asia and South Pacific Design Automation Conference (ASP-DAC). He also received the National Science Foundation Faculty Early Career Development Award for 2000 and the Carnegie Institute of Technology's Ladd Research Award in 2002-2003.

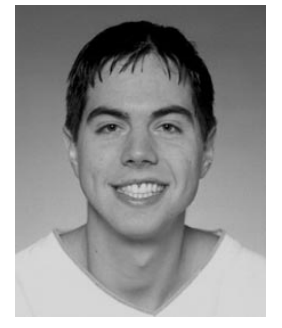

Nicholas H. Zamora received the B.S. degree in electrical engineering and computer science with honors from the University of California, Berkeley, in 2001 and the M.S. degree in electrical and computer engineering from Carnegie Mellon University, Pittsburgh, PA, in 2003. He is working toward the Ph.D. degree at Carnegie Mellon University.

$\mathrm{He}$ is currently working as an SRC graduate fellow, focusing on formal methods for systemlevel design. His previous research interests included supporting development of the Ptolemy project, software for modeling, simulating, and designing concurrent, real-time, embedded systems. His current research interests include ambient intelligent systems.

Mr. Zamora held a UCB Chancellor's Scholarship and received the Eta Kappa Nu Lifetime Achievement Award in 2001.

Phillip Stanley-Marbell (Student Member, IEEE) received the B.Sc. and M.Sc. degrees from Rutgers University, New Brunswick, NJ, in 1999 and 2001, respectively. He is currently working toward the Ph.D. degree at Carnegie Mellon University, Pittsburgh, PA.

His research interests include energy-aware microarchitectures, virtual machines, and fault-tolerant systems.

Mr. Stanley-Marbell is a Student Member of the Association for Computing Machinery.

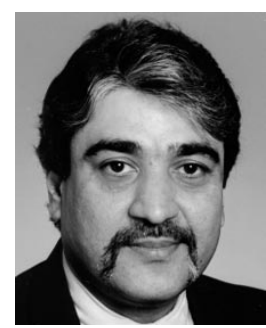

Pradeep K. Khosla (Fellow, IEEE) received the B.Tech. degreee (with honors) from IIT, Kharagpur, India, in 1980 and the M.S. and $\mathrm{Ph} . D$. degrees from Carnegie-Mellon University, Pittsburgh, PA, in 1984 and 1986, respectively.

$\mathrm{He}$ is currently the Philip and Marsha Dowd Professor of Engineering and Robotics, Head of both Electrical and Computer Engineering Department, and Information Networking Institute, and Founding Director of the Center for Computer and Communication Security at Carnegie Mellon University. His research interests are in the areas of internet-enabled distributed and composable simulations, collaborating autonomous systems, agent-based architectures for embedded systems, software composition and reconfigurable software for real-time embedded systems, distributed robotic systems, distributed information systems, and intelligent instruments for biomedical applications.

Dr. Khosla is a recipient of the Inlaks Foundation Fellowship in 1982, the Carnegie Institute of Technology Ladd aAward for excellence in research in 1989, two NASA Tech Brief Awards (1992 and 1993), the ASEE 1999 George Westinghouse Award for Education, the Siliconindia Leadership award for Excellence in Academics and Technology in 2000, and the W. Wallace McDowell Award. He was appointed a Distinguished Lecturer for the IEEE Robotics and Automation Society for 1998-2002. 


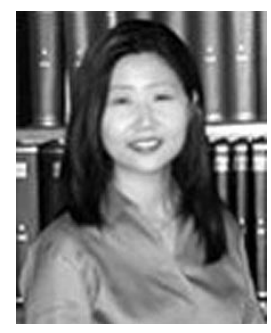

Sungmee Park received the M.S. degree from the Georgia Institute of Technology, Atlanta, in 1995, the M.F.A. degree from Georgia State University, Atlanta, in 1993, and the B.S. degree from Songsim University, Seoul, South Korea, in 1987.

In 1995, she joined the School of Textile \& Fiber Engineering at the Georgia Institute of Technology as a Research Associate and has been working on product design and development projects, including specialty fabrics for the Atlanta Ballet and the smart shirt. The fabric produced for the Atlanta Ballet has been premiered in productions of the ballet. Another significant contribution has been to the realization of the world's first wearable motherboard, also known as the smart shirt. This invention was featured in a Special Issue of Life entitled "Medical Miracles for the New Millennium" (Fall 1998) as "One of the 21 Breakthroughs That Could Change Your Life in the 21st Century." In November 2001, Time named the smart shirt one of the "Best Inventions of the Year 2001."

Ms. Park received Honorable Mention in the upholstery fabric design competition organized by the Carnegie Foundation in 1992.

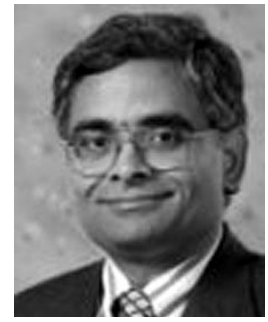

Sundaresan Jayaraman received the Ph.D. degree from North Carolina State University, Raleigh, in 1984, and the M.Tech. and B.Tech. degrees from the University of Madras, Madras, India, in 1978 and 1976, respectively.

In the early 1980s, he was involved in the design and development of TK!Solver, the first equation-solving program from Software Arts, Inc. Before joining Georgia Tech in 1985 he worked as a Product Manager at Software Arts and at Lotus Development Corporation, Cambridge, MA. He is currently Professor of Textile Engineering at the Georgia Institute of Technology in Atlanta, Georgia. He and his research students have made significant contributions in the following areas: 1) enterprise architecture and modeling methodologies for information systems; 2) engineering design of intelligent textile structures and processes; 3 ) design and development of knowledge-based systems (KBS) for textiles and apparel; and 4) multimedia educational systems. His group's research has led to the realization of the world's first Wearable Motherboard-the smart shirt.

Prof. Jayaraman is a recipient of the 1989 NSF Presidential Young Investigator Award. In 1994, he was elected a fellow of the Textile Institute (U.K.). In 2000, he received the Georgia Technology Research Leader Award from the state of Georgia.

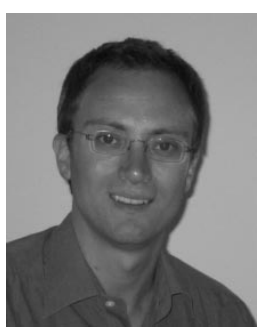

Stefan Jung (Member, IEEE) was born in Germany in 1972. He received the Diploma and Dr.Ing. degrees in electrical engineering from the University of Dortmund, Dortmund, Germany, in 1996 and 2000, respectively.

From 1996 to 1999 he worked on capacitive fingerprint sensors with on-chip signal processing at the Microelectronics Department of Siemens Corporate Technology, Munich, Germany, supported by an Ernst von Siemens scholarship. In 1999, he joined the Emerging Technologies Laboratory within the Corporate Research Department of Infineon Technologies, Munich, Germany. He was engaged in the fields of integrated circuit design, novel sensor and display architectures, and smart wearable electronic devices. He is currently working as Senior Project Manager in the Wearable Technology Solutions project.

Dr. Jung is a Member of the Circuits and Systems Society. He is also a Member of the Association of German Electrical Engineers (VDE) and the German Information Technology Society (ITG).

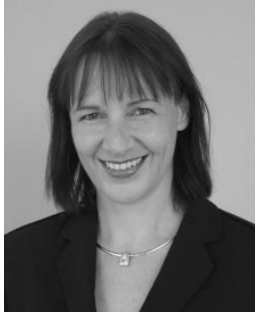

Christl Lauterbach received the Assistant degree in communication engineering from Siemens School, Munich, Germany, in 1977.

She was with the Corporate Research Laboratories of Siemens AG in Munich, Germany, working in the photonic group on technology and device development of infrared emitting diodes and fast photoreceivers. In 1995, she joined the microelectronics IC group, where she was engaged in CMOS basic circuit design, adiabatic circuits, as well as the development of fuse and antifuse structures. Since 1999, she has been a Senior Staff Engineer for Emerging Technologies at Infineon's Corporate Research, Munich, Germany, and is working as Project Manager for smart textiles. She has authored or coauthored some 50 scientific papers. Her main interests in wearable electronics are microelectronic/textile integration and ambient energy conversion.

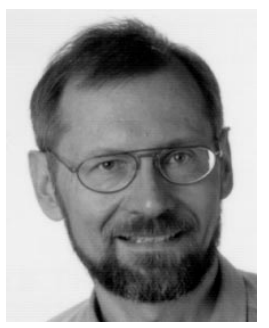

Werner Weber (Senior Member, IEEE) was born in Ruhstorf, Germany, in 1952. He received the Dipl. Phys. degree from the Technische Universität, Munich, Germany, in 1976 and the Dr.rer.nat. degree from the Ludwig-Maximilians-Universität, Munich, Germany, in 1981.

In 1981 he was on a one-year assignment at the IBM Thomas J. Watson Research Center, Yorktown Heights, NY, where he worked in the field of semiconductor thin films. Since 1983 he has been with the Research Laboratories of Siemens AG and Infineon Technologies, Munich, Germany. He was engaged in MOS device physics and basic circuit design and has managed projects on technology-related circuits in advanced memories. At present, he is responsible for the Laboratory on Emerging Technologies at Infineon's Corporate Research, which addresses various topics in connection with ambient intelligence such as wearable electronics, smart textiles, ubiquitous sensor networks, and distributed low-cost electronics. He has authored or coauthored over 150 scientific papers.

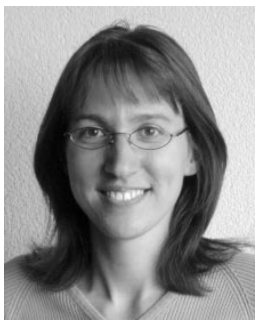

Tünde Kirstein received the M.S. degree in clothing technology from the University of Applied Sciences, Hamburg, Germany, in 1996 and the Ph.D. degree from the Department of Mechanical Engineering, Dresden University of Technology, Dresden, Germany, in 2001

From 1995 to 1997, she was a CAD Engineer with Th. Braun, Hamburg, Germany. From 1996 to 1997 , she was a Lecturer for clothing technology at the Fashion Design Academy Hamburg, Germany. From 1997 to 2001, she was with the Institute of Textile and Clothing Technology, Dresden, Germany. In 2001, she joined the Wearable Computing Laboratory of the Swiss Federal Institute of Technology, Zürich, Switzerland, where she is in charge of the Smart Textiles Group. Her main research interest is the integration of electronic functionality into textiles and clothing.

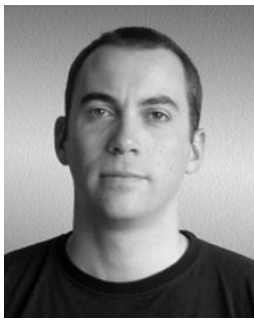

Didier Cottet (Member, IEEE) received the M.S. degree in electrical engineering and the $\mathrm{Ph} . \mathrm{D}$. degree from the Swiss Federal Institute of Technology (ETH), Zürich, Switzerland, in 1997 and 2003, respectively.

He joined the Electronics Laboratory, ETH Zürich, in 1998 as a Teaching and Research Assistant in the High-Density Packaging Group. His research interests include characterization and modeling of thin-film and laminate substrates for high density packaging, and technology optimization for high yield microwave and millimeter-wave system integration. 


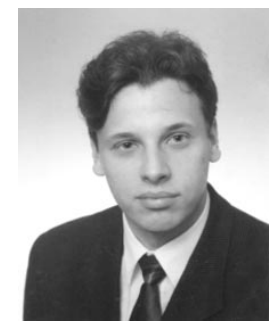

Janusz Grzyb (Member, IEEE) was born in Suwalki, Poland, on May 26, 1972. He received the M.S. degree in electrical engineering from the Technical University, Gdansk, Poland in 1997, where he majored in microelectronics and CMOS analog design. $\mathrm{He}$ is currently working toward the Ph.D. degree at the Swiss Federal Institute of Technology (ETH), Zürich, Switzerland.

Since joining the High-Density Packaging Group at the Electronics Laboratory, ETH Zürich, in 1998, he has been involved in multichip module (MCM)-based packaging and system-in-package solutions. His main interests include high frequency and microwave measurement, design, modeling and simulation of passive structures, development for MCM and related technologies, and analysis and design of CMOS and BiCMOS analog and mixed integrated circuits.

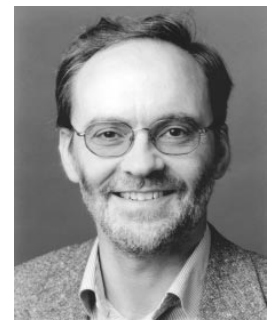

Gerhard Tröster (Member, IEEE) received the M.S. degree in electrical engineering from the Technical University, Karlsruhe, Germany, in 1978 and the Ph.D. degree in electrical engineering from the Technical University, Darmstadt, Germany, in 1984.

During the eight years he spent at Telefunken Corporation, Berlin, Germany, he was responsible for various national and international research projects focused on key components for ISDN and digital mobile phones. He is currently a Professor and Head of the Electronics Laboratory, Swiss Federal Institute of Technology (ETH), Zürich, Switzerland. His field of research includes wearable computing, reconfigurable systems, signal processing, mechatronics, and electronic packaging. He authored and coauthored more than 100 articles and holds five patents. In 1997, he cofounded the spinoff company u-blox AG, a Swiss-based GPS technology provider.

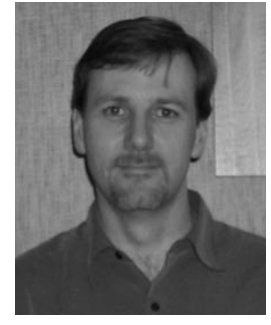

Mark Jones (Member, IEEE) received the B.S. degree in computer science from Clemson University, Clemson, SC, in 1986 and the Ph.D. degree in computer science from Duke University, Durham, NC, in 1990.

He was a member of the technical staff at Argonne National Laboratory and a faculty member at the University of Tennessee. He is currently an Associate Professor in the Bradley Department of Electrical and Computer Engineering, Virginia Tech, Blacksburg. His research interests include high-performance scientific computing, configurable computing, sensor networks, and e-textiles.

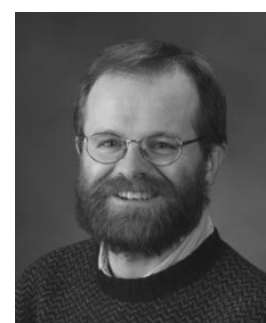

Tom Martin (Member, IEEE) received the B.S degree in electrical engineering with a minor in VLSI systems engineering from the University of Cincinnati, Cincinnati, OH in 1992 and the M.S. and Ph.D. degrees in electrical and computer engineering from Carnegie Mellon University, Pittsburgh, PA, in 1994 and 1999, respectively.

$\mathrm{He}$ is an Assistant Professor in the Bradley Department of Electrical and Computer Engineering, Virginia Tech, Blacksburg. His areas of research include wearable computing, low-power systems, electronic textiles, and mobile computing.

Dr. Martin is a Member of the IEEE Computer Society, the Association for Computing Machinery, and Eta Kappa Nu. He received a National Science Foundation Graduate Research Fellowship from 1993 to 1996.

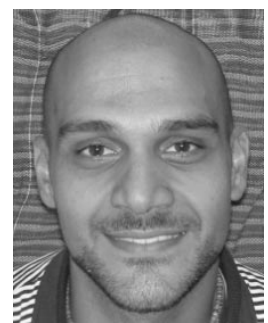

Zahi Nakad (Student Member, IEEE) received the B.E. degree in computer and communication engineering from the American University, Beirut, Lebanon, in 1998 and the M.S. degree in computer engineering from Virginia Tech, Blacksburg, in 2000. He is working toward the $\mathrm{Ph} . \mathrm{D}$. degree in computer engineering in the Bradley Department of Electrical and Computer Engineering, Virginia Tech

Since 1999, he has been a Graduate Research Assistant in the Configurable Computing Lab at Virginia Tech. His research interests include computer architecture, electronic textiles, configurable computing, and analog/digital circuit design. 